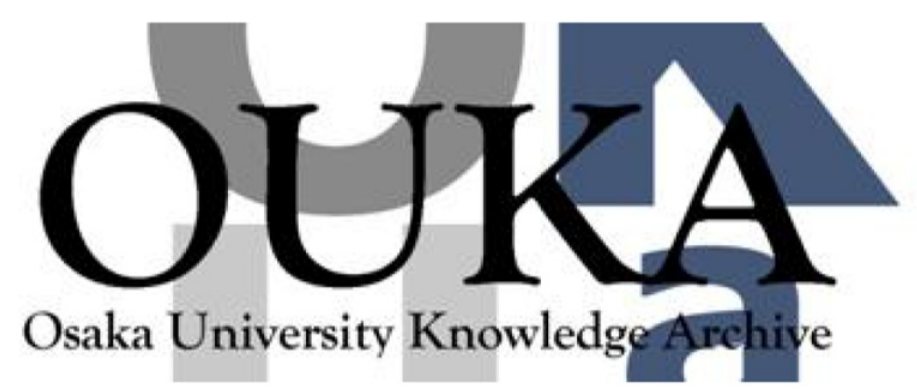

\begin{tabular}{|c|c|}
\hline Title & $\begin{array}{l}\text { Studies of closed/open mirror symmetry for } \\
\text { quintic threefolds through log mixed Hodge } \\
\text { theory }\end{array}$ \\
\hline Author (s) & Usui, Sampei \\
\hline Citation & $\begin{array}{l}\text { Recent advances in Hodge theory : period } \\
\text { domains, algebraic cycles, and arithmetic. } \\
\text { p.134-p. } 164\end{array}$ \\
\hline Issue Date & 2016 \\
\hline oaire:version & VoR \\
\hline URL & https://hdl. handle. net/11094/73378 \\
\hline rights & $\begin{array}{l}\text { @Cambridge University Press } 2016 \text {. This } \\
\text { publication is in copyright. Subject to } \\
\text { statutory exception and to the provisions of } \\
\text { relevant collective licensing agreements, no } \\
\text { reproduction of any part may take place without } \\
\text { the written permission of Cambridge University } \\
\text { Press. }\end{array}$ \\
\hline Note & \\
\hline
\end{tabular}

Osaka University Knowledge Archive : OUKA

https://ir. Library. osaka-u. ac. jp/

Osaka University 


\title{
5
}

\section{Studies of closed/open mirror symmetry for quintic threefolds through $\log$ mixed Hodge theory}

\author{
Sampei Usui
}

Dedicated to Kazuhiro Konno and dedicated to James D. Lewis on his sixtieth birthday

\begin{abstract}
We correct the definitions and descriptions of the integral structures in [30]. The previous flat basis in [ibid] is characterized by the Frobenius solutions and is integral in the first approximation by mean of the graded quotients of monodromy filtration, but it is not integral in the strict sense. In this article, we use $\hat{\Gamma}$-integral structure of Iritani in [7] for A-model. Using this precise version, we study open mirror symmetry for quintic threefolds through log mixed Hodge theory, especially the recent result on Néron models for admissible normal functions with non-torsion extensions in the joint work [14] with K. Kato and C. Nakayama. We understand asymptotic conditions as values in the fiber over a base point on the boundary of $S^{\log }$.
\end{abstract}

\section{Contents}

0 . Introduction

1. Log mixed Hodge theory

1.1. Category $\mathcal{B}(\log )$

1.2. Ringed space $\left(S^{\log }, \mathcal{O}_{S}^{\log }\right)$

1.3. Toric variety

1.4. Local systems on $\left(S^{\log }, \mathcal{O}_{S}^{\text {log }}\right)$

1.5. Graded polarized log mixed Hodge structure

1.6. Nilpotent orbit

1.7. Moduli of log mixed Hodge structures of specified type

1.8. Néron model for admissible normal function

2. Quintic threefolds

2.1. Quintic mirror family

2010 Mathematics Subject Classification. Primary 14C30; Secondary 14D07, 32G20. Partially supported by JSPS. KAKENHI (B) No. 23340008. 
2.2. Picard-Fuchs equation on B-model of mirror $V^{\circ}$

2.3. A-model of quintic $V$

2.4. Integral structure

2.5. Correspondence table

2.6. Proofs of results in 2.5

3. Proof of Theorem 0.4 .1

3.1. Proof of Theorem 0.4 .1 over $\log$ disc $S$

3.2. Proofs of (1) and (2) in Theorem 0.4.1 over log point $p_{0}$

3.3. Discussions on geometries and local systems

\section{Introduction}

In a series of joint works with $\mathrm{K}$. Kato and C. Nakayama, we are constructing a fundamental diagram which consists of various kind of partial compactifications of classifying space of mixed Hodge structures and their relations. We try to understand mirror symmetry in this framework of the fundamental diagram. In this paper, we first complete the insufficient results 3.5-3.6 in the previous paper of Usui [30] (see Remark in 2.6 below), and then study open mirror symmetry for quintic threefolds through log mixed Hodge theory, especially the fine moduli of log Hodge structures and Néron models over it.

\subsection{Fundamental Diagram}

For a classifying space $D$ of Hodge structures of specified type, we have

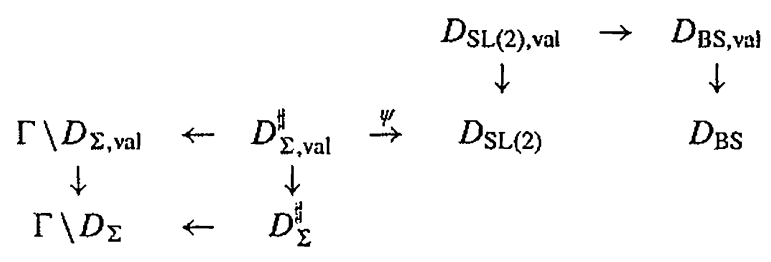

( $\Gamma$ is a monodromy group) in pure case: [15], [16], [17]. For mixed case, we should extend to an amplified diagram: [9], [10], [12], [13], continuing.

\subsection{Mirror symmetry for quintic threefolds}

Let $V$ be a quintic threefold in $\mathbf{P}^{4}$ and $V_{\psi}^{\circ}$ be its mirror family (cf. [1], Sect. 4.2). For simplicity, we denote the family $V_{\psi}^{\circ}$ simply by $V^{\circ}$ if there would be no confusions.

Mirror symmetry for the A-model of quintic threefold $V$ and the B-model of its mirror $V^{\circ}$ was predicted by Candelas-de la Ossa-Green-Parks in the famous paper [2]. We recall two styles of the theorem 0.2.1 and 0.2.2 
below. Every statement in the present paper is near the large radius point $q_{0}$ of the complexified Kähler moduli $\mathcal{K M}(V)$ and the maximally unipotent monodromy point $p_{0}$ of the complex moduli $\mathcal{M}\left(V^{\circ}\right)$.

Let $t:=y_{1} / y_{0}, u:=t / 2 \pi i$ be the canonical parameters and $q:=e^{t}=e^{2 \pi i u}$ be the canonical coordinate for B-model in 2.2 below and the respective ones for A-model in 2.3 below.

The following theorem is due to Givental [5] and Lian-Liu-Yau [20].

Theorem 0.2.1. (Potential). The potentials of the two models coincide: $\Phi_{\mathrm{GW}}^{V}(t)=\Phi_{\mathrm{GM}}^{V^{\circ}}(t)$.

Morrison [22] formulated the following style Theorem 0.2 .2 and proved the theorem except integral structure. Iritani [7] defined $\hat{\Gamma}$-integral structure for A-model and proved the theorem completely for wider objects.

Theorem 0.2.2. (Variation of Hodge structure). The isomorphism ( $q_{0} \in$ $\overline{\mathcal{K M}}(V)) \leftarrow\left(p_{0} \in \overline{\mathcal{M}}\left(V^{\circ}\right)\right)$ of neighborhoods of the compactifications, by the canonical coordinates $q=\exp (2 \pi i u)$, lifts to an isomorphism, over the punctured neighborhoods $\mathcal{K} \mathcal{M}(V) \leftarrow \mathcal{M}\left(V^{\circ}\right)$, of polarized $\mathbf{Z}$-variations of Hodge structure with a specified section

$$
\left(\mathcal{H}^{V}, S, \nabla^{\text {even }}, \mathcal{H}_{\mathrm{Z}}^{V}, F ; T^{3}\right) \leftarrow\left(\mathcal{H}^{V^{\circ}}, Q, \nabla^{\mathrm{GM}}, \mathcal{H}_{\mathrm{Z}}^{V^{\circ}}, F ; \tilde{\Omega}\right) .
$$

Our Theorem 0.2.3 below is equivalent to Theorem 0.2.1 and Theorem 0.2.2 by a $\log$ version [17], Theorem 2.5.14 of the nilpotent orbit theorem of Schmid [27] (this part of [30] is valid).

Theorem 0.2.3. (Log Hodge structure, Log pperiod map). The isomorphism $\left(q_{0} \in \overline{\mathcal{K} M}(V)\right) \stackrel{\leftarrow}{\leftarrow}\left(p_{0} \in \overline{\mathcal{M}}\left(V^{\circ}\right)\right)$ of neighborhoods of the compactifications uniquely lifts to an isomorphism of B-model log variation of polarized Hodge structure with a specified section $\tilde{\Omega}$ for $V^{\circ}$ and A-model log variation of polarized Hodge structure with a specified section $T^{3}$ for $V$, whose restriction over the punctured $\mathcal{K M}(V) \leftarrow \mathcal{M}\left(V^{\circ}\right)$ coincides with the isomorphism of variations of polarized Hodge structure with specified sections in Theorem 0.2.2.

This rephrases as follows. Let $\sigma$ be the common monodromy cone, transformed by a level structure into End of a reference fiber of the local system, for the A-model and for the B-model. Then, we have a commutative diagram of horizontal log period maps

$$
\begin{gathered}
\left(q_{0} \in \overline{\mathcal{K} \mathcal{M}}(V)\right) \stackrel{\sim}{\longleftarrow}\left(p_{0} \in \overline{\mathcal{M}}\left(V^{\circ}\right)\right) \\
\searrow \\
\left(\left[\sigma, \exp \left(\sigma_{\mathrm{C}}\right) F_{0}\right] \in \Gamma(\sigma)^{\mathrm{gP}} \backslash D_{\sigma}\right)
\end{gathered}
$$


with extensions of specified sections in Theorem 0.2 .2 , where $\left[\sigma, \exp \left(\sigma_{\mathrm{C}}\right) F_{0}\right]$ is the class of the nilpotent orbit, regarded as a boundary point, and $\Gamma(\sigma)^{\mathrm{gP}} \backslash D_{\sigma}$ is the fine moduli of log Hodge structures of specified type which will be explained in Section 1 below.

\subsection{Open mirror symmetry for quintic threefolds}

The following theorem is due to Walcher [31] and Morrison-Walcher [23].

Theorem 0.3.1. (Inhomogenous solutions). The potentials of the two models coincide: Let $\mathcal{L}$ be the Picard-Fuchs differential operator for quintic mirror family (cf. 2.2 below). Let

$$
\mathcal{T}_{A}=\frac{u}{2} \pm\left(\frac{1}{4}+\frac{1}{2 \pi^{2}} \sum_{d o d d} n_{d} q^{d / 2}\right)
$$

be the A-model domainwall tension in [23], where the $n_{d}$ are open Gromov-Witten invariants, and

$$
\mathcal{T}_{B}=\int_{C_{-}}^{C_{+}} \Omega
$$

be the $B$-model domainwall tension, where $C_{ \pm} \subset V^{\circ}$ are the disjoint smooth curves coming from the two conics in $\left\{x_{1}+x_{2}=x_{3}+x_{4}=0\right\} \cap V_{\psi} \subset \mathbf{P}^{4}(\mathbf{C})$ [ibid].

Then

$$
\mathcal{L}\left(y_{0}(z) \mathcal{T}_{A}(z)\right)=\mathcal{L}\left(\mathcal{T}_{B}(z)\right)\left(=\frac{15}{16 \pi^{2}} \sqrt{z}\right) \quad\left(z=\frac{1}{(5 \psi)^{5}}\right)
$$

Concerning this, we have the following observations.

\subsection{Log mixed Hodge structure, Log normal function}

We describe for B-model. The same holds for A-model by $0.2-0.3$ and the correspondence table in 2.5 below.

Put $\mathcal{H}:=\mathcal{H}^{V^{\circ}}$ and $\mathcal{T}:=\mathcal{T}_{B}$. We use $e^{0} \in I^{0,0}, e^{1} \in I^{1,1}$ which are a part of a basis of $\mathcal{H}_{\mathcal{O}}$ respecting the Deligne decomposition at $p_{0}$ (see $2.5(3 \mathrm{~B})$ ) and a part of flat basis $s^{0}=e^{0}, s^{1}=e^{1}-(u-1) e^{0}$ of $\mathcal{H}_{Z}$ (see 2.5 (7B)). To make the local monodromy of $\mathcal{T}$ unipotent, we take a double cover $z^{1 / 2} \mapsto z$. Let $L_{Q}$ be the translated local system comparing to the trivial extension $Q(-2) \oplus$ $\mathcal{H}_{\mathrm{Q}}$ by $\left(0,-\left(\mathcal{T} / y_{0}\right) s^{0}\right)$ in $\mathcal{E} x t^{1}\left(\mathrm{Q}(-2), \mathcal{H}_{\mathrm{Q}}\right)$. Let $J_{L_{\mathrm{Q}}}$ be the Néron model on a neighborhood $S$ of $p_{0}$ in the $z^{1 / 2}$-plane which lies over $L_{Q}$ in [14] (there is a difference of Tate twist). Then, $J_{L_{Q}}=\mathcal{E} x t_{\mathrm{LMH} / S}^{1}(\mathbf{Z}(-2), \mathcal{H})$ (extension group of $\log$ mixed Hodge structures over $S$ ) in the present case (cf. [13], Corollary 6.1 .6 , and 1.8 below), and we have the following theorem. 
Theorem 0.4.1. (Log normal function).

(1) The normalized tension $\mathcal{T} / y_{0}$ is understood as a multi-valued truncated normal function by $\left(\mathcal{T} / y_{0}\right) s^{0}$. Then it lifts and extends uniquely to a single-valued $\log$ normal function $S \rightarrow J_{L_{\mathrm{Q}}}$ so that the corresponding exact sequence $0 \rightarrow \mathcal{H} \rightarrow \tilde{\mathcal{H}} \rightarrow \mathbf{Z}(-2) \rightarrow 0$ of log mixed Hodge structures over $S$ is given by the liftings $1_{\mathbf{Z}}$ and $1_{F}$ in $\tilde{\mathcal{H}}$ of $(2 \pi i)^{-2} \cdot 1 \in \mathbf{Z}(-2)$ respecting the lattice and the Hodge filtration, respectively, which are defined as follows: $1_{\mathrm{Z}}:=\left((2 \pi i)^{-2} \cdot 1,-\left(\mathcal{T} / y_{0}\right) s^{0}\right)$ with $\left(\mathcal{T} / y_{0}\right) s^{0} \in$ $\mathcal{H}_{\mathcal{O}^{\log }}=\left(\mathrm{gr}_{3}^{W}\right)_{\mathcal{O}} \log$, and $1_{F}-1_{\mathrm{Z}}:=\left(\delta\left(\mathcal{T} / y_{0}\right)\right) e^{1}-\left(\mathcal{T} / y_{0}\right) e^{0}$, where $\delta:=$ $2 \pi i q d / d q$.

(2) A splitting of the weight filtration $W$ of the local system $\tilde{\mathcal{H}}_{\mathrm{Z}}$, i.e., a splitting compatible with the monodromy of the local system $\tilde{\mathcal{H}}_{\mathrm{Z}}$, is given by $1_{\mathbf{Z}}^{\text {spl }}=$ $1_{\mathbf{Z}}+s^{1} / 2$, and the $\log$ normal function over it is given by $1_{F}^{s p l}-1_{\mathbf{Z}}^{\text {spl }}=$ $\left(\delta\left(\mathcal{T} / y_{0}\right)\right) e^{1}-\left(\mathcal{T} / y_{0}\right) e^{0}$, where $\delta$ is as in $(1)$.

(3) Theorem 0.3.1 says that the inverse of the normal function in (1) from its image is given by $16 \pi^{2} / 15$ times the Picard-Fuchs differential operator $\mathcal{L}$.

Theorem 0.4.1 is proved in Section 3, and after these proofs some geometric backgrounds are discussed in Section 3.3.

\section{5 .}

The organization of this paper is as follows. Section 1 is a summary of log mixed Hodge theory mainly from [17], [13] and [14], which is used to study mirror symmetry in later sections and also is expected to work as a brief guide of this theory. In Section 2, after preparations including $\hat{\Gamma}$-integral structure in [6] and [7], we give a correspondence table of closed mirror symmetry for quintic threefolds and their mirrors, which is the precision and the expansion of our previous paper [30], Sect. 3. In Section 3, we prove log mixed Hodge theoretic interpretation Theorem 0.4.1. We also give some discussions on the related geometries and local systems in Section 3.3.

Acknowledgments. The author thanks Kazuya Kato and Chikara Nakayama for series of joint works on log Hodge theory, from which he learns a lot and enjoys exciting studies. He thanks Hiroshi Iritani for pointing out insufficient parts in the previous paper [30]. He also thanks Yukiko Konishi and Satoshi Minabe, together with Iritani, for stimulating seminars on the present topic. The author thanks the referees for careful readings and useful comments.

\section{Notation.}

Fix $\Lambda:=\left(H_{0}, W,\left(\langle,\rangle_{w}\right)_{w},\left(h^{p, q}\right)_{p, q}\right)$, where

$H_{0}$ is a free $\mathbf{Z}$-module of finite rank, 
$W$ is an increasing filtration on $H_{0, \mathrm{Q}}:=\mathrm{Q} \otimes H_{0}$,

$\langle,\rangle_{w}$ is a non-degenerate $(-1)^{w}$-symmetric bilinear form on $\mathrm{gr}_{w}^{W}$,

$\left(h^{p, q}\right)_{p, q}$ is a set of Hodge numbers.

$D$ : the classifying space of graded polarized mixed Hodge structures for the data $\Lambda$, consisting of all Hodge filtrations.

$\check{D}$ : the "compact dual" of $D$.

$G_{A}:=\operatorname{Aut}\left(H_{0, A}, W,\left(\langle,\rangle_{w}\right)_{w}\right)$, where $H_{0, A}:=A \otimes H_{0}(A=\mathbf{Z}, \mathbf{Q}, \mathbf{R}, \mathbf{C})$,

$\mathfrak{g}_{A}:=\operatorname{Lie} G_{A}=\operatorname{End}\left(H_{0, A}, W,\left(\langle,\rangle_{w}\right)_{w}\right)(A=\mathbf{Q}, \mathbf{R}, \mathbf{C})$.

\section{Log mixed Hodge theory}

This section is a summary of log mixed Hodge theory from [17], [13], and [14]. We write a general form of these results as a brief guide for future use. Section 1.8 is adapted for the use in Section 3. The corresponding results in [13] and [14] are written in more general settings.

\subsection{Category $\mathcal{B}(\log )$}

Let $S$ be a subset of an analytic space $Z$. The strong topology of $S$ in $Z$ is the strongest one among those topologies on $S$ in which, for any analytic space $A$ and any morphism $f: A \rightarrow Z$ with $f(A) \subset S$ as sets, $f: A \rightarrow S$ is continuous. $S$ is regarded as a local ringed space by the pullback sheaf of $\mathcal{O}_{Z}$.

Let $\mathcal{B}$ be the category of local ringed spaces $S$ over $\mathbf{C}$ which have an open covering $\left(U_{\lambda}\right)_{\lambda}$, satisfying the following condition: For each $\lambda$, there exist an analytic space $Z_{\lambda}$, and a subset $S_{\lambda}$ of $Z_{\lambda}$ such that, as local ringed space over C, $U_{\lambda}$ is isomorphic to an open subset of $S_{\lambda}$ which is endowed with the strong topology in $Z_{\lambda}$ and the inverse image of $\mathcal{O}_{Z_{\lambda}}$.

A $\log$ structure on a local ringed space $S$ is a sheaf of monoids $M$ on $S$ together with a homomorphisim $a: M \rightarrow \mathcal{O}_{S}$ such that $\alpha^{-1} \mathcal{O}_{S}^{\times} \stackrel{\sim}{\rightarrow} \mathcal{O}_{S}^{\times} \cdot f s \log$ structure means, locally on the underlying space, the log structure has a chart which is finitely generated, integral and saturated.

Let $\mathcal{B}(\log )$ be the category of objects of $\mathcal{B}$ endowed with an fs log structure.

A log analytic space is called $\log$ smooth if, locally, it is isomorphic to an open set of a toric variety endowed with the canonical log structure. A $\log$ manifold is a $\log$ local ringed space over $\mathbf{C}$ which has an open covering $\left(U_{\lambda}\right)_{\lambda}$ satisfying the following condition: For each $\lambda$, there exist a log smooth fs $\log$ analytic space $Z_{\lambda}$, a finite subset $I_{\lambda}$ of global $\log$ differential 1 -forms $\Gamma\left(Z_{\lambda}, \omega_{Z_{\lambda}}^{1}\right)$, and an isomorphism of log local ringed spaces over $\mathrm{C}$ between $U_{\lambda}$ and an open subset of $S_{\lambda}:=\left\{z \in Z_{\lambda} \mid\right.$ the image of $I_{\lambda}$ in the stalk $\omega_{z}^{1}$ is zero $\}$ in the strong topology in $Z_{\lambda}$. 


\subsection{Ringed space $\left(S^{\log }, \mathcal{O}_{S}^{\log }\right)$}

The ringed space $\left(S^{\log }, \mathcal{O}_{S}^{\text {log }}\right)$ was defined for fs $\log$ schemes by K. Kato and $\mathrm{C}$. Nakayama in [8]. It was generalized for the category $\mathcal{B}(\log )$ in [15].

Let $S \in \mathcal{B}(\log )$. As a set define

$S^{\log }:=\left\{(s, h) \mid s \in S, h: M_{s}^{\mathrm{gp}} \rightarrow \mathbf{S}^{1}\right.$ homomorphism s.t. $h(u)=u /|u|$ if $u \in \mathcal{O}_{S, s}^{\times}$.

Endow $S^{\log }$ with the weakest topology such that the following two maps are continuous.

(1) $\tau: S^{\log } \rightarrow S,(s, h) \mapsto s$.

(2) For any open set $U \subset S$ and any $f \in \Gamma\left(U, M^{\mathrm{gp}}\right), \tau^{-1}(U) \rightarrow \mathbf{S}^{1},(s, h) \mapsto$ $h\left(f_{s}\right)$.

Then, $\tau$ is proper and surjective with fiber $\tau^{-1}(s)=\left(\mathbf{S}^{1}\right)^{r(s)}$, where $r(s)$ is the rank of $\left(M^{\mathrm{gP}} / \mathcal{O}_{S}^{\times}\right)_{s}$ which varies with $s \in S$.

Define a sheaf $\mathcal{L}$ on $S^{\text {log }}$ as the fiber product:

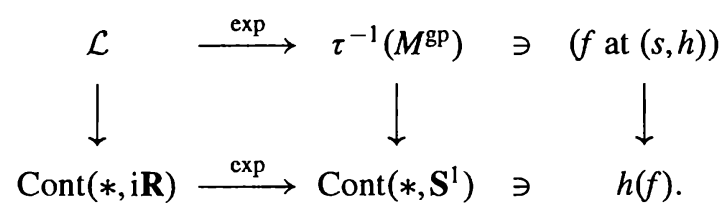

Let $l: \tau^{-1}\left(\mathcal{O}_{S}\right) \rightarrow \mathcal{L}$ be a morphism induced from

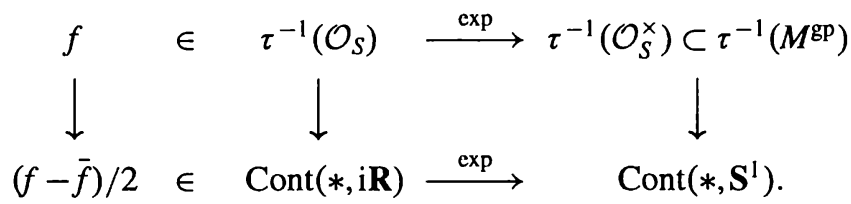

Define

$$
\mathcal{O}_{S}^{\log }:=\frac{\tau^{-1}\left(\mathcal{O}_{S}\right) \otimes \operatorname{Sym}_{\mathbf{Z}}(\mathcal{L})}{\left(f \otimes 1-1 \otimes \imath(f) \mid f \in \tau^{-1}\left(\mathcal{O}_{S}\right)\right)} .
$$

Thus $\tau:\left(S^{\log }, \mathcal{O}_{S}^{\log }\right) \rightarrow\left(S, \mathcal{O}_{S}\right)$ is a morphism of ringed spaces over $\mathbf{C}$. For $s \in S$ and $t \in S^{\log }$ lying over $s$, let $t_{j} \in \mathcal{L}_{t}(1 \leq j \leq r(s))$ be elements such that their images in $\left(M^{\mathrm{gP}} / \mathcal{O}_{S}^{\times}\right)_{s}$ of $\exp \left(t_{j}\right)$ form a basis. Then, $\mathcal{O}_{S, t}^{\mathrm{log}}=\mathcal{O}_{S, s}\left[t_{j}(1 \leq j \leq\right.$ $r(s))]$ is a polynomial ring.

\subsection{Toric variety}

Toric varieties offer typical examples of $S^{\text {log }}$ and also they are building blocks of fine moduli spaces of log mixed Hodge structures.

Let $\sigma \subset \mathfrak{g}_{\mathbf{R}}$ be a nilpotent cone, i.e., a sharp cone, $\sigma \cap(-\sigma)=\{0\}$, generated by a finite number of mutually commutative nilpotent elements. Assume that the cone generators of $\sigma$ can be taken from $g_{\mathbf{Q}}$. Let $\Gamma$ be a subgroup of $G_{\mathbf{Z}}$. 
Define a monoid $\Gamma(\sigma):=\Gamma \cap \exp (\sigma)$ and the dual monoid $P(\sigma):=\Gamma(\sigma)^{\vee}=$ $\operatorname{Hom}(\Gamma(\sigma), \mathbf{N})$. Define a toric variety and a torus by

$$
\begin{aligned}
\operatorname{toric}_{\sigma}: & =\operatorname{Spec}(\mathbf{C}[P(\sigma)])(\mathbf{C})=\operatorname{Hom}\left(P(\sigma), \mathbf{C}^{\text {mult }}\right) \supset \text { torus }_{\sigma}: \\
& =\operatorname{Hom}\left(P(\sigma)^{\mathrm{gp}}, \mathbf{C}^{\times}\right),
\end{aligned}
$$

where $\mathbf{C}^{\text {mult }}$ is $\mathbf{C}$ regarded as a monoid by multiplication and $P(\sigma)^{\mathrm{gP}}$ is the group generated by the monoid $P(\sigma)$. The exponential sequence $0 \rightarrow \mathbf{Z} \rightarrow$ $\mathbf{C} \rightarrow \mathbf{C}^{\times} \rightarrow 1$ induces the universal covering of the torus

$$
0 \rightarrow \operatorname{Hom}\left(P(\sigma)^{\mathrm{gp}}, \mathbf{Z}\right) \rightarrow \operatorname{Hom}\left(P(\sigma)^{\mathrm{gp}}, \mathbf{C}\right) \stackrel{\mathrm{e}}{\rightarrow} \operatorname{Hom}\left(P(\sigma)^{\mathrm{gp}}, \mathbf{C}^{\times}\right) \rightarrow 1,
$$

where $\operatorname{Hom}\left(P(\sigma)^{\mathrm{gp}}, \mathbf{Z}\right)=\Gamma(\sigma)^{\mathrm{gp}}$ is considered as the fundamental group of torus $_{\sigma}$, and $\mathbf{e}(z \otimes \gamma):=e^{2 \pi i z} \otimes \gamma\left(z \in \mathbf{C}, \gamma \in \Gamma(\sigma)^{\mathrm{gp}}=\operatorname{Hom}\left(P(\sigma)^{\mathrm{gP}}, \mathbf{Z}\right)\right)$.

Fix the above cone $\sigma$. For a face $\rho$ of $\sigma$, define $\tilde{P}(\rho):=\left\{l \in P(\sigma)^{\mathrm{gp}} \mid l(\rho) \geq 0\right\}$. Then we have an open covering

$$
\text { toric }_{\sigma}=\operatorname{Spec}(\mathbf{C}[P(\sigma)])(\mathbf{C})=\bigcup_{\rho \prec \sigma} \operatorname{Spec}(\mathbf{C}[\tilde{P}(\rho)])(\mathbf{C}) .
$$

We now recall a stratification. Fix the above cone $\sigma$ and let $\rho$ be a face of the cone $\sigma$. Then, we have a homomorphism $P(\rho) \lambda P(\sigma)$ and hence a morphism toric $_{\rho} \rightarrow$ toric $_{\sigma}$. The origin $0_{\rho} \in$ toric $_{\rho}$ is the monoid homomorphism $P(\sigma) \rightarrow$ $C^{\text {mult }}$ sending 1 to 1 and all the other elements of $P(\rho)$ to 0 , which is sent to a point of toric ${ }_{\sigma}$ by the above morphism. Then, as a set, we have a stratification into torus orbits

$$
\text { toric }_{\sigma}=\left\{\mathbf{e}(z) 0_{\rho} \mid \rho \prec \sigma, z \in \sigma_{\mathbf{C}} /\left(\rho_{\mathbf{C}}+\log \Gamma(\sigma)^{\mathrm{gP}}\right)\right\} .
$$

Here $\mathbf{e}(c \log \gamma):=\mathbf{e}(c \otimes \gamma)=e^{2 \pi i c} \otimes \gamma\left(c \in \mathbf{C}, \gamma \in \Gamma(\sigma)^{\mathrm{gp}}\right)$.

For $S:=$ toric $_{\sigma}$, the polar coordinate $\mathbf{R}_{\geq 0} \times \mathbf{S}^{1} \rightarrow \mathbf{R}_{\geq 0} \mathbf{S}^{\prime}=\mathbf{C}$ induces $\tau$ : $S^{\log } \rightarrow S$ as

$$
\begin{aligned}
& \tau: S^{\log }=\operatorname{Hom}\left(P(\sigma), \mathbf{R}_{\geq 0}^{\text {mult }} \times \operatorname{Hom}\left(P(\sigma), \mathbf{S}^{\mathrm{l}}\right)\right. \\
& =\left\{\left(\mathbf{e}(i y) 0_{\rho}, \mathbf{e}(x)\right) \mid \rho \prec \sigma, x \in \sigma_{\mathbf{R}} /\left(\rho_{\mathbf{R}}+\log \Gamma(\sigma)^{\mathrm{gP}}\right), y \in \sigma_{\mathbf{R}} / \rho_{\mathbf{R}}\right\} \\
& \rightarrow S=\operatorname{Hom}\left(P(\sigma), \mathbf{C}^{\text {mult }}\right), \\
& \tau\left(\mathbf{e}(i b) 0_{\rho}, \mathbf{e}(a)\right)=\mathbf{e}(a+i b) 0_{\rho} .
\end{aligned}
$$

Since $0 \rightarrow \rho_{\mathbf{R}} / \log \Gamma(\rho)^{\mathrm{gP}} \rightarrow \sigma_{\mathbf{R}} / \log \Gamma(\sigma)^{\mathrm{gp}} \rightarrow \sigma_{\mathbf{R}} /\left(\rho_{\mathbf{R}}+\log \Gamma(\sigma)^{\mathrm{gP}}\right) \rightarrow 0$ is exact, the fiber of $\tau$, as a set, is described as

$$
\tau^{-1}\left(\mathbf{e}(a+i b) 0_{\rho}\right)=\left\{\left(\mathbf{e}(i b) 0_{\rho}, \mathbf{e}(a+x)\right) \mid x \in \rho_{\mathbf{R}} / \log \Gamma(\rho)^{\mathrm{gp}}\right\} \simeq\left(\mathbf{S}^{1}\right)^{r},
$$

where $r=r(\rho):=\operatorname{rank} \rho$ varies with $\rho \prec \sigma$. 
Let $H_{\sigma}=\left(H_{\sigma, \mathrm{Z}}, W,\left(\langle,\rangle_{w}\right)_{w}\right)$ be the canonical local system endowed with the weight filtration and the polarizations on graded quotients on $S^{\log }$, which are given by the representation $\pi_{1}\left(S^{\log }\right)=\Gamma(\sigma)^{\mathrm{gp}} \subset G_{\mathbf{Z}}=$ $\operatorname{Aut}\left(H_{0}, W,\left(\langle,\rangle_{w}\right)_{w}\right)$.

\subsection{Local systems on $\left(S^{\log }, \mathcal{O}_{S}^{\log }\right)$}

We recall three results about local systems on $\left(S^{\log }, \mathcal{O}_{S}^{\log }\right) \in \mathcal{B}(\log )$ from [17], Sect. 2.3.

Let $L$ be a locally constant sheaf of abelian groups on $S^{\log }$. For $s \in S$ and $t \in S^{\log }$ lying over $s$, we call the action of $\pi_{1}\left(s^{\log }\right)=\pi_{1}\left(\tau^{-1}(s)\right)$ on $L_{t}$ the local monodromy of $L$ at $t$. We say the local monodromy of $L$ is unipotent if the local monodromy of $L$ at $t$ is unipotent for any $t \in S^{\log }$.

Let $s \in S$. Let $\left(q_{j}\right)_{1 \leq j \leq n}$ be a finite family of elements of $M_{S, s}^{\mathrm{gp}}$ whose image in $\left(M_{S}^{\mathrm{gp}} / \mathcal{O}_{S}^{\times}\right)_{s}$ is a free basis, and let $\left(\gamma_{j}\right)_{1 \leq j \leq n}$ be the dual basis of $\pi_{1}\left(s^{\log }\right)$, that is $\left[\gamma_{j}, q_{k}\right]=(2 \pi i) \delta_{j k}$ where [, ] is the pairing given by $\pi_{1}\left(s^{\log }\right) \simeq$ $\operatorname{Hom}\left(M_{s}^{\mathrm{gp}} / \mathcal{O}_{s}^{\times}, \mathbf{Z}\right)$.

Let $L$ be a locally constant sheaf on $S^{\log }$ of free $\mathbf{Z}$-modules of finite rank. Let $s \in S$ and $t \in \tau^{-1}(s)$, and assume that the local monodromy of $L$ at $t$ is unipotent. For a fixed $t$, we denote $L_{0}$ the constant sheaf on $S^{\log }$ with fiber $L_{t}$. Let $L_{0, \mathbf{Q}}=\mathbf{Q} \otimes_{A} L_{0}$, and let $N_{j}: L_{0, \mathbf{Q}} \rightarrow L_{0, \mathbf{Q}}$ be the endomorphism of constant sheaf which is induced by the logarithm of the monodromy action of $\gamma_{j}$ on the stalk $L_{t}$ of the locally constant sheaf $L$. Lift $q_{j}$ in $\Gamma\left(S, M_{S}^{\mathrm{gp}}\right)$ (by replacing $S$ by an open neighborhood of $s$ ), and let

$$
\xi=\exp \left(\sum_{j=1}^{n}(2 \pi i)^{-1} \log \left(q_{j}\right) \otimes N_{j}\right): \mathcal{O}_{S}^{\log } \otimes_{\mathbf{Q}} L_{0, \mathbf{Q}} \stackrel{\sim}{\rightarrow} \mathcal{O}_{S}^{\log } \otimes_{\mathbf{Q}} L_{0, \mathbf{Q}}
$$

Note that the operator $\xi$ depends on the choices of the branches of $\log \left(q_{j}\right)$ in $\mathcal{O}_{S}^{\log }$ locally on $S^{\log }$, but that the subsheaf $\xi^{-1}\left(1 \otimes L_{0}\right)$ of $\mathcal{O}_{S}^{\log } \otimes_{\mathbf{Z}} L_{0}$ is independent of the choices and hence is defined globally on $S^{\log }$.

The following proposition shows that the locally constant sheaf $L$ is embedded in $\mathcal{O}_{S}^{\log } \otimes L_{0}$.

Proposition 1.4.1. ([17], Prop. 2.3.2). Let the situation be as above. If we replace $S$ by some open neighborhood of $s$, we have an isomorphism of $\mathcal{O}_{S}^{\log }$-modules

$$
v: \mathcal{O}_{S}^{\log } \otimes_{A} L \stackrel{\sim}{\rightarrow} \mathcal{O}_{S}^{\log } \otimes_{A} L_{0}
$$

satisfying the following condition (1).

(1) The restriction of $v$ to $L=1 \otimes L$ induces an isomorphism of locally constant sheaves $v: L \stackrel{\sim}{\rightarrow} \xi^{-1}\left(1 \otimes L_{0}\right)$. 
If we take suitable branches $\log \left(q_{j}\right)_{t, 0}$ in $\mathcal{O}_{S, t}^{\log }$ of the germs $\log \left(q_{j}\right)_{t}$ at $t$ $(1 \leq j \leq n)$, we can take an isomorphism $v$ which satisfies above (1) and also the following (2).

(2) The branch $\xi_{t, 0}$ of the germ $\xi_{t}$, defined by the fuxed branches $\log \left(q_{j}\right)_{t, 0}$ of the germs $\log \left(q_{j}\right)_{t}$, satisfies $v(1 \otimes v)=\xi_{t, 0}^{-1}(1 \otimes v)$ for any $v \in L_{t}=L_{0}$.

The following proposition yields a log Hodge theoretic understanding [ibid, Sect. 2.3.6] of the canonical extension of Deligne in [3].

Proposition 1.4.2. ([17], Prop. 2.3.3). Let $S \in \mathcal{B}(\log )$ and let L be a locally constant sheaf of finite dimensional $\mathbf{C}$-vector spaces on $S^{\log }$.

(i) If the local monodromy of $L$ is unipotent, the $\mathcal{O}_{S}$-module $\mathcal{M}:=$ $\tau_{*}\left(\mathcal{O}_{S}^{\log } \otimes_{\mathrm{C}} L\right)$ is locally free of finite rank, and we have an isomorphism $\mathcal{O}_{S}^{\log } \otimes_{\mathcal{O}_{S}} \mathcal{M} \stackrel{\sim}{\rightarrow} \mathcal{O}_{S}^{\log } \otimes_{\mathrm{C}} L$.

(ii) Conversely, assume that there are a locally free $\mathcal{O}_{S}$-module $\mathcal{M}$ of finite rank on $S$ and an isomorphism of $\mathcal{O}_{S}^{\log }$-modules $\mathcal{O}_{S}^{\log } \otimes_{\mathcal{O}_{S}} \mathcal{M} \simeq \mathcal{O}_{S}^{\log } \otimes_{\mathrm{C}} L$. Then the local monodromy of $L$ is unipotent and $\mathcal{M} \stackrel{\sim}{\rightarrow} \tau_{*}\left(\mathcal{O}_{S}^{\log } \otimes_{\mathrm{C}} L\right)$.

Proposition 1.4.3. ([17], Prop. 2.3.4). Let $S \in \mathcal{B}(\log )$, let $L$ be a locally constant sheaf on $S^{\log }$ of free $\mathbf{Q}$-modules of finite rank. Assume that the local monodromy of $L$ is unipotent.

(i) There exists a unique $\mathbf{Q}$-homomorphism

$$
\mathcal{N}: L \rightarrow\left(M_{S}^{\mathrm{gp}} / \mathcal{O}_{S}^{\times}\right) \otimes L
$$

satisfying the following condition (1).

(1) For any $s \in S$, any $t \in S^{\log }$ lying over $s$, and any $\gamma \in \pi_{1}\left(s^{\log }\right)$, if $h_{\gamma}$ : $\left(M_{S}^{\mathrm{gp}} / \mathcal{O}_{S}^{\times}\right)_{s} \rightarrow \mathbf{Z}$ denotes the homomorphism corresponding to $\gamma$ by $\pi_{1}\left(s^{\log }\right) \simeq$ $\operatorname{Hom}\left(M_{s}^{\mathrm{gp}} / \mathcal{O}_{s}^{\times}, \mathbf{Z}\right)$, the composition $L_{t} \stackrel{\mathcal{N}}{\rightarrow}\left(M_{S}^{\mathrm{gp}} / \mathcal{O}_{S}^{\times}\right)_{s} \otimes L_{t} \stackrel{h_{\gamma}}{\rightarrow} L_{t}$ coincides with the logarithm of the action of $\gamma$ on $L_{t}$.

(ii) Assume that $S$ is an $f$ s log point $\{s\}$. Let

$$
\mathcal{N}^{\prime}: L \rightarrow \omega_{s}^{1} \otimes L
$$

be the composition of $\mathcal{N}$ and the $\mathbf{Q}$-linear map $M_{s}^{\mathrm{gP}} / \mathcal{O}_{s}^{\times} \otimes L \rightarrow \omega_{s}^{1} \otimes L, f \otimes v \mapsto$ $(2 \pi i)^{-1} d \log (f) \otimes v$, and let $1 \otimes \mathcal{N}^{\prime}: \mathcal{O}_{s}^{\log } \otimes L \rightarrow \omega_{s}^{1, \log } \otimes L$ be the $\mathcal{O}_{s}^{\log }$-linear homomorphism induced by $\mathcal{N}^{\prime}$. Let $\mathcal{M}:=H^{0}\left(s^{\log }, \mathcal{O}_{s}^{\log } \otimes L\right)=\tau_{*}\left(\mathcal{O}_{s}^{\log } \otimes L\right)$. Then the restriction $\mathcal{M} \rightarrow \omega_{s}^{1} \otimes_{\mathrm{C}} \mathcal{M}$ of $d \otimes 1_{L}: \mathcal{O}_{s}^{\log } \otimes L \rightarrow \omega_{s}^{1, \log } \otimes L$ coincides with the restriction of $1 \otimes \mathcal{N}^{\prime}$ to $\mathcal{M}$.

$\mathcal{N}$ in the above proposition is described as follows. Assume $L=\xi^{-1}\left(1 \otimes L_{0}\right)$ as in the first proposition. Then $\mathcal{N}\left(\xi^{-1}(1 \otimes v)\right):=\sum_{j=1}^{n} q_{j} \otimes \xi^{-1}\left(1 \otimes N_{j} v\right)$ for $v \in L_{0}$. 


\subsection{Graded polarized log mixed Hodge structure}

Let $S \in \mathcal{B}(\log )$. A pre-graded polarized log mixed Hodge structure on $S$ is a tuple $H=\left(H_{\mathbf{Z}}, W,\left(\langle,\rangle_{w}\right)_{w}, H_{\mathcal{O}}\right)$ consisting of a local system of $\mathbf{Z}$-free modules $H_{\mathbf{Z}}$ of finite rank on $S^{\log }$, an increasing filtration $W$ of $H_{\mathbf{Q}}:=\mathbf{Q} \otimes H_{\mathbf{Z}}$, a non-degenerate $(-1)^{w}$-symmetric Q-bilinear form $\langle,\rangle_{w}$ on $\mathrm{gr}_{w}^{W}$, a locally free $\mathcal{O}_{S}$-module $H_{\mathcal{O}}$ on $S$, a specified isomorphism $\mathcal{O}_{S}^{\text {log }} \otimes_{\mathbf{Z}} H_{\mathbf{Z}} \simeq \mathcal{O}_{S}^{\log } \otimes_{\mathcal{O}_{S}} H_{\mathcal{O}}$ (log Riemann-Hilbert correspondence), and a specified decreasing filtration $F H_{\mathcal{O}}$ of $H_{\mathcal{O}}$ such that $F^{p} H_{\mathcal{O}}$ and $H_{\mathcal{O}} / F^{p} H_{\mathcal{O}}$ are locally free. Put $F^{p}:=$ $\mathcal{O}_{S}^{\log } \otimes_{\mathcal{O}_{S}} F^{p} H_{\mathcal{O}}$. Then $\tau_{*} F^{p}=F^{p} H_{\mathcal{O}}$. For each integer $w$, the orthogonality condition $\left\langle F^{p}\left(\mathrm{gr}_{w}^{W}\right), F^{q}\left(\mathrm{gr}_{w}^{W}\right)\right\rangle_{w}=0(p+q>w)$ is imposed.

A pre-graded polarized log mixed Hodge structure on $S$ is a graded polarized log mixed Hodge structure on $S$ if its pullback to each $s \in S$ is a graded polarized log mixed Hodge structure on $s$ in the following sense.

Let $\left(H_{\mathbf{Z}}, W,\left(\langle,\rangle_{w}\right)_{w}, H_{\mathcal{O}}\right)$ be a pre-graded polarized log mixed Hodge structure on a log point $s$. It is a graded polarized log mixed Hodge structure if it satisfies the following three conditions.

(1) (Admissibility). For each logarithm $N$ of the local monodromy of the local system $\left(H_{\mathbf{R}}, W,\left(\langle,\rangle_{w}\right)_{w}\right)$, there exists a $W$-relative $N$-filtration $M(N, W)$.

(2) (Griffiths transversality). For any integer $p, \nabla F^{p} \subset \omega_{s}^{1, \log } \otimes F^{p-1}$ is satisfied, where $\omega_{s}^{1, \log }$ is the sheaf of $\mathcal{O}_{s}^{\log }$-module of log differential 1-forms on $\left(s^{\log }, \mathcal{O}_{s}^{\log }\right)$, and $\nabla=d \otimes 1_{H_{\mathbf{Z}}}: \mathcal{O}_{s}^{\log } \otimes H_{\mathbf{Z}} \rightarrow \omega_{s}^{1, \log } \otimes H_{\mathbf{Z}}$ is the log Gauss-Manin connection.

(3) (Positivity). For a point $t \in s^{\log }$ and a $\mathbf{C}$-algebra homomorphism $a$ : $\mathcal{O}_{s, t}^{\log } \rightarrow \mathbf{C}$, define a filtration $F(a):=\mathbf{C} \otimes_{\mathcal{O}_{s, t}^{\log }} F_{t}$ on $H_{\mathbf{C}, t}$. Then, $\left(H_{\mathbf{Z}, t}\left(\mathrm{gr}_{w}^{W}\right),\langle,\rangle_{w}\right.$, $F(a))$ is a polarized Hodge structure of weight $w$ in the usual sense if $a$ is sufficiently twisted, i.e., for $\left(q_{j}\right)_{1 \leq j \leq n} \subset M_{s}$ inducing generators of $M_{s} / \mathcal{O}_{s}^{\times}$, $\left|\exp \left(a\left(\log q_{j}\right)\right)\right| \ll 1$ for any $j$.

\subsection{Nilpotent orbit}

Let $\sigma \subset \mathfrak{g}_{\mathbf{R}}$ be a nilpotent cone (see 1.3). A subset $Z \subset \check{D}$ is $\sigma$-nilpotent orbit if the following (1)-(4) hold for $F \in Z$.

(1) $Z=\exp \left(\sigma_{\mathrm{C}}\right) F$.

(2) For any $N \in \sigma$, there exists $W$-relative $N$-filtration $M(N, W)$.

(3) For any $N \in \sigma$ any $p, N F^{p} \subset F^{p-1}$.

(4) If $N_{1}, \ldots, N_{n}$ generate $\sigma$ and $y_{j} \gg 0$ for any $j$, then $\exp \left(\sum_{j} i y_{j} N_{j}\right) F \in D$.

A weak fan $\Sigma$ in $\mathrm{g}_{\mathbf{Q}}$ is a set of nilpotent cones in $\mathfrak{g}_{\mathbf{R}}$, defined over $\mathbf{Q}$, which satisfies the following three conditions.

(5) Every $\sigma \in \Sigma$ is admissible relative to $W$.

(6) If $\sigma \in \Sigma$ and $\tau \prec \sigma$, then $\tau \in \Sigma$. 
(7) If $\sigma, \sigma^{\prime} \in \Sigma$ have a common interior point and if there exists $F \in \check{D}$ such that $(\sigma, F)$ and $\left(\sigma^{\prime}, F\right)$ generate nilpotent orbits, then $\sigma=\sigma^{\prime}$.

Let $\Sigma$ be a weak fan and $\Gamma$ be a subgroup of $G_{\mathbf{Z}} . \Sigma$ and $\Gamma$ are strongly compatible if the following two conditions are satisfied.

(8) If $\sigma \in \Sigma$ and $\gamma \in \Gamma$, then $\operatorname{Ad}(\gamma) \sigma \in \Sigma$.

(9) For any $\sigma \in \Sigma, \sigma$ is generated by $\log \Gamma(\sigma)$, where $\Gamma(\sigma):=\Gamma \cap \exp (\sigma)$.

\subsection{Moduli of log mixed Hodge structures of type $\Phi$}

Let $\Phi:=(\Lambda, \Sigma, \Gamma)$ be a data consisting of a Hodge data $\Lambda$ (in Notation), a weak fan $\Sigma$ and a subgroup $\Gamma$ of $G_{\mathbf{Z}}$ such that $\Sigma$ and $\Gamma$ are strongly compatible (1.6).

Let $\sigma \in \Sigma$ and $S:=$ toric $_{\sigma}$. Let $H_{\sigma}=\left(H_{\sigma, \mathrm{Z}}, W,\left(\langle,\rangle_{w}\right)_{w}\right)$ be the canonical local system $H_{\sigma, \mathrm{Z}}$ endowed with the weight filtration $W$ and the polarizations $\langle,\rangle_{w}$ on the graded quotients $\operatorname{gr}_{w}^{W}(w \in \mathbf{Z})$ over $S^{\log }$, which is determined by the representation $\Gamma \subset G_{\mathbf{Z}}=\operatorname{Aut}\left(H_{0}, W,\left(\langle,\rangle_{w}\right)_{w}\right)$.

Let $\breve{E}_{\sigma}:=$ toric $_{\sigma} \times \check{D}$. The universal pre-graded polarized log mixed Hodge structure $H$ on $\check{E}_{\sigma}$ is given by $H_{\sigma}$ together with the isomorphism $\mathcal{O}_{\breve{E}_{\sigma}}^{\log } \otimes_{\mathbf{Z}}$ $H_{\sigma, \mathbf{Z}}=\mathcal{O}_{\check{E}_{\sigma}}^{\log } \otimes \mathcal{O}_{\check{E}_{\sigma}} H_{\mathcal{O}}(1.5)$, where $H_{\mathcal{O}}:=\mathcal{O}_{\check{E}_{\sigma}} \otimes H_{0}$ is the free $\mathcal{O}_{\check{E}_{\sigma}}$-module coming from that on $\check{D}$ endowed with the universal Hodge filtration $F$.

Let $E_{\sigma}:=\left\{x \in \check{E}_{\sigma}|H|_{x}\right.$ is a graded polarized log mixed Hodge structure on $\left.x\right\}$. Note that slits appear in $E_{\sigma}$ because of $\log$-pointwise Griffiths transversality 1.5 (2) and positivity $1.5(3)$, or equivalently $1.6(3)$ and $1.6(4)$ respectively.

As a set, define $D_{\Sigma}:=\{(\sigma, Z) \mid$ nilpotent orbit, $\sigma \in \Sigma, Z \subset \check{D}\}$. Let $\sigma \in \Sigma$. Assume that $\Gamma$ is neat. A structure as an object of $\mathcal{B}(\log )$ on $\Gamma \backslash D_{\Sigma}$ is introduced by a diagram:

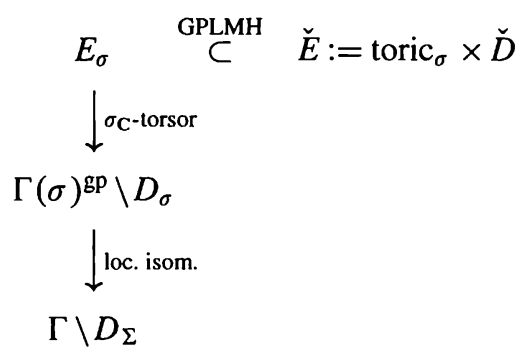

The action of $h \in \sigma_{\mathbf{C}}$ on $\left(\mathbf{e}(a) 0_{\rho}, F\right) \in E_{\sigma}$ is $\left(\mathbf{e}(h+a) 0_{\rho}, \exp (-h) F\right)$, and the projection is $\left(\mathbf{e}(a) 0_{\rho}, F\right) \mapsto\left[\rho, \exp \left(\rho_{\mathbf{C}}+a\right) F\right]$.

Let $S \in \mathcal{B}(\log )$. A log mixed Hodge structure of type $\Phi$ on $S$ is a pre-graded polarized $\log$ mixed Hodge structure $H=\left(H_{\mathbf{Z}}, W,\left(\langle,\rangle_{w}\right)_{w}, H_{\mathcal{O}}\right)$ endowed with $\Gamma$-level structure $\mu \in H^{0}\left(S^{\log }, \Gamma \backslash \mathcal{I} \operatorname{som}\left(\left(H_{\mathbf{Z}}, W,\left(\langle,\rangle_{w}\right)_{w}\right),\left(H_{0}, W,\left(\langle,\rangle_{w}\right)_{w}\right)\right)\right)$ satisfying the following condition: For any point $s \in S$, any point $t \in \tau^{-1}(s)=$ $s^{\log }$ and any representative $\tilde{\mu}_{t}: H_{\mathrm{Z}, t} \stackrel{\sim}{\rightarrow} H_{0}$, there exists $\sigma \in \Sigma$ such that $\sigma$ 
contains $\tilde{\mu}_{t} \pi_{1}^{+}\left(s^{\log }\right) \tilde{\mu}_{t}^{-1}$ and $\left(\sigma, \tilde{\mu}_{t}\left(\mathbf{C} \otimes_{\mathcal{O}_{S, t}^{\log } F_{t}}\right)\right)$ generates a nilpotent orbit. Here $\pi_{1}^{+}\left(s^{\log }\right):=\operatorname{Image}\left(\operatorname{Hom}\left(\left(M_{S} / \mathcal{O}_{S}^{\times}\right)_{s}, \mathbf{N}\right) \hookrightarrow \pi_{1}\left(s^{\log }\right) \rightarrow \operatorname{Aut}\left(H_{\mathbf{Z}, t}\right)\right)$ is the local monodromy monoid of $H_{\mathbf{Z}}$ at $s$ (cf. [17], Sect. 3.3.2). (Then, the smallest such $\sigma$ exists.)

Theorem 1.7.1. For a given data $\Phi$, we have the following.

(i) $\Gamma \backslash D_{\Sigma} \in \mathcal{B}(\log )$, which is Hausdorff. If $\Gamma$ is neat, $\Gamma \backslash D_{\Sigma}$ is a log manifold. (ii) On $\mathcal{B}(\log ), \Gamma \backslash D_{\Sigma}$ represents a functor $\mathrm{LMH}_{\Phi}$ of log mixed Hodge structures of type $\Phi$.

Log period map. Given $\Phi$. Let $S \in \mathcal{B}(\log )$. Then we have an isomorphism

$$
\begin{aligned}
\mathrm{LMH}_{\Phi}(S) \stackrel{\sim}{\rightarrow} \operatorname{Map}\left(S, \Gamma \backslash D_{\Sigma}\right), \\
\quad H \mapsto\left(S \ni s \mapsto\left[\sigma, \exp \left(\sigma_{\mathbf{C}}\right) \tilde{\mu}_{t}\left(\mathbf{C} \otimes_{\mathcal{O}_{S, t}^{\log }} F_{t}\right)\right]\right)\left(t \in \tau^{-1}(s)\right),
\end{aligned}
$$

which is functorial in $S$.

A log period map is a unified compactification of a period map and a normal function of Griffiths.

The above $\Gamma \backslash D_{\Sigma}$ is the fine moduli of log mixed Hodge structures of type $\Phi$, whose underlying coarse moduli, in the sense of log points, is the set of equivalence classes of all nilpotent orbits of specified type.

\subsection{Néron model for admissible normal function}

We review some results from [14], Theorem 1.3, [13], Section 6.1, and [11], Section 8 adapted to the situation 0.4 in Introduction.

For a pure case $h^{p, q}=1(p+q=3, p, q \geq 0)$ and $h^{p, q}=0$ otherwise, a complete fan is constructed in [17], Section 12.3. For a mixed case $h^{p, q}=1$ (the above $(p, q)$, plus $(p, q)=(2,2))$ and $h^{p, q}=0$ otherwise, over the above fan, a fan of Néron model for given admissible normal function is constructed in [14], Theorem 3.1, and we have a Néron model in the following sense.

Let $S \in \mathcal{B}(\log ), U:=S_{\text {triv }} \subset S$ (consisting of those points with trivial log structure), $H_{(-1)}$ be a polarized variation of Hodge structure of weight -1 (Tate-twisted by 2 for $\mathcal{H}$ in Introduction 0.4 ) on $U$ and $L_{Q}$ be a local system of $\mathbf{Q}$-vector spaces which is an extension of $\mathbf{Q}$ by $H_{(-1), \mathbf{Q}}$. An admissible normal function over $U$ for $H_{(-1)}$ underlain by the local system $L_{\mathbf{Q}}$ can be regarded as an admissible variation of mixed Hodge structure which is an extension of $\mathbf{Z}$ by $H_{(-1)}$ and lies over local system $L_{\mathrm{Q}}$.

For any given unipotent admissible normal function over $U$ as above, $H_{(-1)}$ and $L_{Q}$ extend to a polarized $\log$ mixed Hodge structure on $S$ and a local system on $S^{\mathrm{log}}$, respectively, denoted by the same symbols, and there is a relative log manifold $J_{L_{Q}}$ over $S$ which is strict over $S$ (i.e., endowed with the pullback log 
structure from $S)$ and which represents the following functor on $\mathcal{B} / S^{\circ}\left(S^{\circ} \in \mathcal{B}\right.$ is the underlying space of $S$ ):

$S^{\prime} \mapsto\left\{\mathrm{LMH} H\right.$ on $S^{\prime}$ satisfying $H\left(\mathrm{gr}_{w}^{W}\right)=\left.H_{(w)}\right|_{S^{\prime}}(w=-1,0)$ and $(*)$ below \}/isom.

(*) Locally on $S^{\prime}$, there is an isomorphism $H_{\mathbf{Q}} \simeq L_{\mathbf{Q}}$ on $\left(S^{\prime}\right)^{\log }$ preserving $W$.

Here $H_{(w)} \mid S^{\prime}$ is the pullback of $H_{(w)}$ by the structure morphism $S^{\prime} \rightarrow S^{\circ}$, and $S^{\prime}$ is endowed with the pullback log structure from $S$.

Put $H^{\prime}:=H_{(-1)}$. In the present case, we have $J_{L_{\mathbf{Q}}}=\mathcal{E} x t_{\mathrm{LMH} / S}^{1}\left(\mathbf{Z}, H^{\prime}\right)$ by [13], Corollary 6.1.6. This is the subgroup of $\tau_{*}\left(H_{\mathcal{O}}^{\prime}{ }^{\prime}\right.$ og $\left./\left(F^{0}+H_{\mathbf{Z}}^{\prime}\right)\right)$ restricted by admissibility condition and log-pointwise Griffiths transversality condition ([11], Section 8, cf. 1.5). Let $\tilde{J}_{L_{\mathbf{Q}}}$ be the pullback of $J_{L_{\mathbf{Q}}}$ by $\tau_{*}\left(H_{\mathcal{O}^{\prime l o g}}^{\prime} / F^{0}\right) \rightarrow$ $\tau_{*}\left(H_{\mathcal{O}^{\log }}^{\prime} /\left(F^{0}+H_{\mathbf{Z}}^{\prime}\right)\right)$, and $\bar{J}_{L_{\mathbf{Q}}}$ be the image of $\tilde{J}_{L_{\mathbf{Q}}}$ by $\tau_{*}\left(H_{\mathcal{O}^{\log }}^{\prime} / F^{0}\right) \rightarrow$ $\tau_{*}\left(H_{\mathcal{O}}^{\prime}{ }^{\text {log }} / F^{-1}\right)$. Then, by using the polarization, we have a commutative diagram:

$$
\begin{aligned}
& J_{L_{\mathbf{Q}}}=\mathcal{E} x t_{\mathrm{LMH} / \mathrm{S}}^{1}\left(\mathbf{Z}, H^{\prime}\right) \subset \tau_{*}\left(H_{\mathcal{O}^{\log }}^{\prime} /\left(F^{0}+H_{\mathbf{Z}}^{\prime}\right)\right) \underset{\sim}{\stackrel{\mathrm{pol}}{\longrightarrow}} \tau_{*}\left(\left(F^{0}\right)^{*} / H_{\mathbf{Z}}^{\prime}\right) \\
& \begin{array}{ccccc}
\uparrow & & \uparrow & \uparrow \\
\tilde{J}_{L_{Q}} & \subset & \tau_{*}\left(H_{\mathcal{O}^{l o g}}^{\prime} / F^{0}\right) & \stackrel{\text { pol }}{\longrightarrow} & \tau_{*}\left(\left(F^{0}\right)^{*}\right) \\
\downarrow & \downarrow & & \downarrow \\
\bar{J}_{L_{\mathbf{Q}}} & \subset & \tau_{*}\left(H_{\mathcal{O}^{\log }}^{\prime} / F^{-1}\right) & \stackrel{\text { pol }}{\longrightarrow} & \tau_{*}\left(\left(F^{1}\right)^{*}\right) .
\end{array}
\end{aligned}
$$

\section{Quintic threefolds}

Let $V$ be a quintic threefold in $\mathbf{P}^{4}$ and let $V_{\psi}^{\circ}$ be its mirror family (cf. [1], Sect. 4.2).

In this section, we give a correspondence table of A-model for $V$ and B-model for $V_{\psi}^{\circ}$. This is a precision and an expansion of our previous [30], Sect. 3 by using $\hat{\Gamma}$-integral structure of Iritani [7]. We will use this table in Section 3 below.

\subsection{Quintic mirror family}

Following [21], [23], etc., we briefly recall the construction of the mirror family $V_{\psi}^{\circ}$ by quotient method. Let $V_{\psi}: f:=\sum_{j=1}^{5} x_{j}^{5}-5 \psi \prod_{j=1}^{5} x_{j}=0\left(\psi \in \mathbf{P}^{1}\right)$ be the Dwork pencil of quintics in $\mathbf{P}^{4}$. Let $\mu_{5}$ be the group consisting of the fifth roots of the unity in $\mathbf{C}$. Then the group $G:=\left\{\left(a_{j}\right) \in\left(\mu_{5}\right)^{5} \mid a_{1} \ldots a_{5}=1\right\}$ acts on $V_{\psi}$ by $x_{j} \mapsto a_{j} x_{j}$. Let $V_{\psi}^{\circ}$ be a crepant resolution of quotient singularity of $V_{\psi} / G$ (cf. [21], [23]). Divide further by the action $\left(x_{1}, \ldots, x_{5}\right) \mapsto\left(a^{-1} x_{1}, x_{2}, \ldots, x_{5}\right)$ and $\psi \mapsto a \psi\left(a \in \mu_{5}\right)$. 


\subsection{Picard-Fuchs equation on the mirror $V^{\circ}$}

Let $\Omega$ be a 3-form on $V_{\psi}^{\circ}$ with a $\log$ pole over $\psi=\infty$ induced from

$$
\left(\frac{5}{2 \pi i}\right)^{3} \operatorname{Res}_{V_{\psi}}\left(\frac{\psi}{f} \sum_{j=1}^{5}(-1)^{j-1} x_{j} d x_{1} \wedge \cdots \wedge \widehat{d x}_{j} \wedge \cdots \wedge d x_{5}\right) .
$$

Let $z:=1 /(5 \psi)^{5}$ and $\theta:=z d / d z$. Let

$$
\mathcal{L}:=\theta^{4}-5 z(5 \theta+1)(5 \theta+2)(5 \theta+3)(5 \theta+4)
$$

be the Picard-Fuchs differential operator for $\Omega$, i.e., $\mathcal{L} \Omega=0$ via the Gauss-Manin connection $\nabla$. There are three special points of the complex moduli:

$z=0:$ maximally unipotent monodromy point,

$z=\infty$ : Gepner point,

$z=1 / 5^{5}:$ conifold point.

At $z=0$, the Picard-Fuchs differential equation $\mathcal{L} y=0$ has the indicial equation $\rho^{4}=0$ ( $\rho$ is indeterminate), i.e., maximally unipotent. By the Frobenius method, we have a basis of solutions $y_{j}(z)(0 \leq j \leq 3)$ as follows. Let

$$
\tilde{y}(-z ; \rho):=\sum_{n=0}^{\infty} \frac{\prod_{m=1}^{5 n}(5 \rho+m)}{\prod_{m=1}^{n}(\rho+m)^{5}}(-z)^{n+\rho}
$$

be a solution of $\mathcal{L}(\tilde{y}(-z ; \rho))=\rho^{4}(-z)^{\rho}$, and let

$\tilde{y}(-z ; \rho)=y_{0}(z)+y_{1}(z) \rho+y_{2}(z) \rho^{2}+y_{3}(z) \rho^{3}+\cdots, \quad y_{j}(z):=\left.\frac{1}{j !} \frac{\partial^{i} \tilde{y}(-z ; \rho)}{\partial \rho^{j}}\right|_{\rho=0}$ be the Taylor expansion at $\rho=0$. Then, $y_{j}(0 \leq j \leq 3)$ form a basis of homogeneous solutions for the linear differential equation $\mathcal{L} y=0$. We have

$$
\begin{aligned}
& y_{0}=f_{0}=\sum_{n=0}^{\infty} \frac{(5 n) !}{(n !)^{5}} z^{n}, \\
& y_{1}=f_{0} \log z+f_{1}=y_{0} \log z+5 \sum_{n=1}^{\infty} \frac{(5 n) !}{(n !)^{5}}\left(\sum_{j=n+1}^{5 n} \frac{1}{j}\right) z^{n}, \\
& 2 ! y_{2}=f_{0}(\log z)^{2}+2 f_{1} \log z+f_{2}, \\
& 3 ! y_{3}=f_{0}(\log z)^{3}+3 f_{1}(\log z)^{2}+3 f_{2} \log z+f_{3},
\end{aligned}
$$

where all $f_{j}$ are holomorphic functions in $z$ with $f_{0}(0)=1$ and $f_{j}(0)=0$ for $j>0$.

Define the canonical parameters by $t:=y_{1} / y_{0}, u:=t / 2 \pi i$, and the canonical coordinate by $q:=e^{t}=e^{2 \pi i u}$ which is a specific chart of the log structure given by the divisor $(z=0)$ of a disc in $\mathbf{P}^{1}$ and gives a mirror map. 
Write $z=z(q)$ which is holomorphic in $q$. Then we have

$$
\log z=2 \pi i u-\frac{5}{y_{0}(z(q))} \sum_{n=1}^{\infty} \frac{(5 n) !}{(n !)^{5}}\left(\sum_{j=n+1}^{5 n} \frac{1}{j}\right) z(q)^{n} .
$$

The Gauss-Manin potential of $V_{z}^{\circ}$ is

$$
\Phi_{\mathrm{GM}}^{V^{\circ}}=\frac{5}{2}\left(\frac{y_{1}}{y_{0}} \frac{y_{2}}{y_{0}}-\frac{y_{3}}{y_{0}}\right)
$$

Let $\tilde{\Omega}:=\Omega / y_{0}$ and $\delta:=2 \pi i q d / d q=d u$. Then, the Yukawa coupling at $z=0$ is

$$
Y:=-\int_{V^{\circ}} \tilde{\Omega} \wedge \nabla_{\delta} \nabla_{\delta} \nabla_{\delta} \tilde{\Omega}=\frac{5}{\left(1+5^{5} z\right) y_{0}(z)^{2}}\left(\frac{q d z}{z d q}\right)^{3} .
$$

\subsection{A-model of quintic $V$}

Let $V$ be a general quintic hypersurface in $\mathbf{P}^{4}$. Let $H$ be the cohomology class of a hyperplane section of $V$ in $\mathbf{P}^{4}, K(V)=\mathbf{R}_{>0} H$ be the Kähler cone of $V$, and $u$ be the coordinate of $\mathbf{C H}$. Put $t:=2 \pi i u$. A complexified Kähler moduli is defined as

$$
\mathcal{K} \mathcal{M}(V):=\left(H^{2}(V, \mathbf{R})+i K(V)\right) / H^{2}(V, \mathbf{Z}) \stackrel{\sim}{\rightarrow} \Delta^{*}, \quad u H \mapsto q:=e^{2 \pi i u} .
$$

Let $C \in H_{2}(V, \mathbf{Z})$ be the homology class of a line on $V$.

For $\beta=d C \in H_{2}(V, \mathbf{Z})$, define $q^{\beta}:=q^{d}$. The Gromov-Witten potential of $V$ is defined as

$$
\Phi_{\mathrm{GW}}^{V}:=\frac{1}{6} \int_{V}(2 \pi i u H)^{3}+\sum_{0 \neq \beta \in H_{2}(V, \mathrm{Z})} N_{d} q^{\beta}=\frac{5}{6}(2 \pi i)^{3} u^{3}+\sum_{d>0} N_{d} q^{d}
$$

Here the Gromov-Witten invariant $N_{d}$ is

$$
\begin{gathered}
\bar{M}_{0,0}\left(\mathbf{P}^{4}, d\right) \stackrel{\pi_{1}}{\leftarrow} \bar{M}_{0,1}\left(\mathbf{P}^{4}, d\right) \stackrel{e_{1}}{\rightarrow} \mathbf{P}^{4}, \\
N_{d}:=\int_{\bar{M}_{0,0}\left(\mathbf{P}^{4}, d\right)} c_{5 d+1}\left(\pi_{1 *} e_{1}^{*} \mathcal{O}_{\mathbf{P}^{4}}(5)\right) .
\end{gathered}
$$

Note that $N_{d}=0$ if $d \leq 0$. Let $N_{d}=\sum_{k \mid d} n_{d / k} k^{-3}$. Then $n_{d / k}$ is the instanton number. ( $n_{l}$ here is different from $n_{l}$ in Theorem 0.3.1.)

The differentials of $\Phi=\Phi_{\mathrm{GW}}^{V}$ are computed easily:

$$
\frac{d \Phi}{d u}=\frac{5}{2}(2 \pi i)^{3} u^{2}+(2 \pi i) \sum_{d>0} N_{d} d q^{d}, \quad \frac{d^{2} \Phi}{d u^{2}}=5(2 \pi i)^{3} u+(2 \pi i)^{2} \sum_{d>0} N_{d} d^{2} q^{d}
$$

\subsection{Integral structure}

As we stated in Introduction, we consider everything near the large radius point $q_{0}$ and the maximally unipotent monodromy point $p_{0}$. Let $S$ be a neighborhood 
disc of $q_{0}$ (resp. $\left.p_{0}\right)$ in $\overline{\mathcal{K} \mathcal{M}}(V)$ (resp. $\overline{\mathcal{M}}\left(V^{\circ}\right)$ ) for A-model of $V$ (resp. for B-model of $V^{\circ}$ ), and let $S^{*}$ be $S \backslash\left\{q_{0}\right\}$ (resp. $S \backslash\left\{p_{0}\right\}$ ) for A-model (resp. B-model) (see 2.2, 2.3). Endow $S$ with the $\log$ structure associated to the divisor $S \backslash S^{*}$.

The B-model variation of Hodge structure $\mathcal{H}^{V^{\circ}}$ is the usual variation of Hodge structure arising from the smooth projective family $f: X \rightarrow S^{*}$ of the quintic mirrors over the punctured neighborhood of $p_{0}$. Its integral structure is the usual one $\mathcal{H}_{\mathbf{Z}}^{V^{\circ}}=R^{3} f_{*} \mathbf{Z}$. This is compatible with the monodromy weight filtration $M$ around $p_{0}$. Define $M_{k, \mathbf{Z}}:=M_{k} \cap \mathcal{H}_{\mathbf{Z}}^{V^{\circ}}$ for all $k$.

For the A-model $\mathcal{H}^{V}$ on $S^{*}$, the locally free sheaf on $S^{*}$, the Hodge filtration, and the monodromy weight filtration $M$ around $q_{0}$ are given by $\mathcal{H}_{\mathcal{O}}^{V}:=$ $\mathcal{O}_{S^{*}} \otimes\left(\bigoplus_{0 \leq p \leq 3} H^{2 p}(V)\right), F^{p}:=\mathcal{O}_{S^{*}} \otimes H^{\leq 2(3-p)}(V)$, and $M_{2 p}:=H^{\geq 2(3-p)}(V)$, respectively. Iritani defined $\hat{\Gamma}$-integral structure in more general setting in [7], Definition 3.6. In the present case, it is characterized as follows. Let $H$ and $C$ be a hyperplane section and a line on $V$, respectively. Then, in the present case, a basis of the $\hat{\Gamma}$-integral structure is given by $\left\{s(\mathcal{E}) \mid \mathcal{E}\right.$ is $\left.\mathcal{O}_{V}, \mathcal{O}_{H}, \mathcal{O}_{C}, \mathcal{O}_{\mathrm{pt}}\right\}$ [ibid, Example 6.18], where $s(\mathcal{E})$ is a unique $\nabla^{\text {even }}$-flat section satisfying an asymptotic condition

$$
s(\mathcal{E}) \sim(2 \pi i)^{-3} e^{-2 \pi i u H} \cdot \hat{\Gamma}\left(T_{V}\right) \cdot(2 \pi i)^{\operatorname{dcg} / 2} \operatorname{ch}(\mathcal{E})
$$

at the large radius point $q_{0}$ when $\operatorname{Im}(u) \rightarrow \infty$ for each fixed $\operatorname{Re}(u)$. Here, for the Chern roots $c\left(T_{V}\right)=\prod_{j=1}^{3}\left(1+\delta_{j}\right)$, the Gamma class $\hat{\Gamma}\left(T_{V}\right)$ is defined by

$$
\begin{aligned}
\hat{\Gamma}\left(T_{V}\right):=\prod_{j=1}^{3} \Gamma\left(1+\delta_{j}\right) & =\exp \left(-\gamma c_{1}(V)+\sum_{k \geq 2}(-1)^{k}(k-1) ! \zeta(k) \operatorname{ch}_{k}\left(T_{V}\right)\right) \\
& =\exp \left(\zeta(2) \operatorname{ch}_{2}\left(T_{V}\right)-2 \zeta(3) \operatorname{ch}_{3}\left(T_{V}\right)\right)
\end{aligned}
$$

where $\gamma$ is the Euler constant, and $\left.\operatorname{deg}\right|_{H^{2 p}(V)}:=2 p$. The important point is that this class $\hat{\Gamma}\left(T_{V}\right)$ plays the role of a "square root" of the Todd class in Hirzebruch-Riemann-Roch ([6], 1; [7], 1, (13)). Denote this $\hat{\Gamma}$-integral structure by $\mathcal{H}_{\mathbf{Z}}^{V}$. This is compatible with the monodromy weight filtration $M$ and we define $M_{k, \mathrm{Z}}:=M_{k} \cap \mathcal{H}_{\mathrm{Z}}^{V}$ for all $k$. The above asymptotic relation is actually computed as

$$
\begin{aligned}
& s^{0}:=s\left(\mathcal{O}_{\mathrm{pt}}\right)=\frac{1}{5} H^{3}, \\
& s^{1}:=s\left(\mathcal{O}_{C}\right)=\frac{1}{5}(2 \pi i)^{-1} H^{2}+\frac{1}{5}(-u+1) H^{3}, \\
& s^{2}:=s\left(\mathcal{O}_{H}\right) \sim(2 \pi i)^{-2} H+\frac{5}{2}(2 \pi i)^{-1}\left(-u-\frac{1}{2}\right) H^{2}+\left(\frac{1}{2} u^{2}+\frac{1}{2} u+\frac{7}{12}\right) H^{3},
\end{aligned}
$$




$$
\begin{aligned}
s^{3}:= & s\left(\mathcal{O}_{V}\right) \sim(2 \pi i)^{-3}-(2 \pi i)^{-2} u H+(2 \pi i)^{-1}\left(\frac{1}{2} u^{2}+\frac{5}{12}\right) H^{2} \\
& +\left(-\frac{1}{6} u^{3}-\frac{5}{12} u+\frac{5 i \zeta(3)}{\pi^{3}}\right) H^{3} .
\end{aligned}
$$

Fixing an isomorphism of VHS in (2) in Introduction, we also use $s^{p}$ for the corresponding $\nabla$-flat integral basis for the $\mathrm{B}$-model $\mathcal{H}_{\mathrm{Z}}^{V^{\circ}}$ (vanishing cycles are used for B-model in [7], Theorems 6.9, 6.10, Example 6.18).

In both A-model case and B-model case, the integral structures $\mathcal{H}_{\mathrm{Z}}^{V}$ and $\mathcal{H}_{\mathrm{Z}}^{V^{\circ}}$ on $S^{*}$ extend to the local systems of $\mathbf{Z}$-modules over $S^{\log }$ ([24]; [17], Proposition 2.3.5), still denoted $\mathcal{H}_{\mathbf{Z}}^{V}$ and $\mathcal{H}_{\mathbf{Z}}^{V^{\circ}}$, respectively.

Consider a diagram:

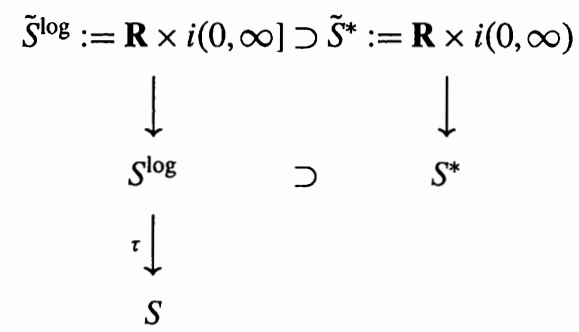

The coordinate $u$ of $\tilde{S}^{*}$ extends over $\tilde{S}^{\log }$. Fix base points as $u_{0}=0+i \infty \epsilon$ $\tilde{S}^{\log } \mapsto b:=\overline{0}+i \infty \in S^{\log } \mapsto q=0 \in S$, where $q=0$ corresponds to $q_{0}$ for A-model and $p_{0}$ for B-model. Note that fixing a base point $u=u_{0}$ on $\tilde{S}^{\log }$ is equivalent to fixing a base point $b$ on $S^{\log }$ and also a branch of $(2 \pi i)^{-1} \log q$.

Let $B:=\mathcal{H}_{\mathbf{Z}}^{V}\left(u_{0}\right)=\mathcal{H}_{\mathbf{Z}}^{V}(b)$ for A-model and $B:=\mathcal{H}_{\mathbf{Z}}^{V^{\circ}}\left(u_{0}\right)=\mathcal{H}_{\mathbf{Z}}^{V^{\circ}}(b)$ for B-model.

\subsection{Correspondence table}

We use the mirror theorems in Introduction $0.2-0.3$. Put $\Phi:=\Phi_{\mathrm{GW}}^{V}=\Phi_{\mathrm{GM}}^{V^{\circ}}$ and fix an isomorphisim of VHS in 0.2.2 in Introduction (cf. 2.4).

(1A) Polarization of A-model of $V$.

$$
S(\alpha, \beta):=(-1)^{p}(2 \pi i)^{3} \int_{V} \alpha \cup \beta \quad\left(\alpha \in H^{p, p}(V), \beta \in H^{3-p, 3-p}(V)\right) .
$$

(1B) Polarization of $B$-model of $V^{\circ}$.

$$
Q(\alpha, \beta):=(-1)^{3(3-1) / 2} \int_{V^{\circ}} \alpha \cup \beta=-\int_{V^{\circ}} \alpha \cup \beta \quad\left(\alpha, \beta \in H^{3}\left(V^{\circ}\right)\right) .
$$

(2A) Z-basis compatible with monodromy weight filtration.

Let $B:=\mathcal{H}_{\mathbf{Z}}^{V}\left(u_{0}\right)=\mathcal{H}_{\mathbf{Z}}^{V}(b)$ be as in Section 2.4. Let $b^{3}:=s^{3}\left(u_{0}\right)=s\left(\mathcal{O}_{V}\right)\left(u_{0}\right)$, $b^{2}:=s^{2}\left(u_{0}\right)=s\left(\mathcal{O}_{H}\right)\left(u_{0}\right), b^{1}:=s^{1}\left(u_{0}\right)=s\left(\mathcal{O}_{C}\right)\left(u_{0}\right)$ and $b^{0}:=s^{0}\left(u_{0}\right)=$ $s\left(\mathcal{O}_{\mathrm{pt}}\right)\left(u_{0}\right)$ be the basis of the fiber $B$ at $u_{0}$ coming from $\nabla$-flat integral basis in 2.4 . 
The endomorphism of $B_{\mathbf{Q}}:=\mathbf{Q} \otimes B$ coming from the monodromy logarithm coincides with the cup product with $-2 \pi i H$ where $H$ is a hyperplane section of $V$ ([7], Definition 3.6; cf. Proposition 1.4.3). Hence the above basis is compatible with the monodromy weight filtration $M$.

(2B) Z-basis compatible with monodromy weight filtration.

Let $B:=\mathcal{H}_{\mathrm{Z}}^{V^{\circ}}\left(u_{0}\right)=\mathcal{H}_{\mathrm{Z}}^{V^{\circ}}(b)$, and $b^{0}, b^{1}, b^{2}, b^{3}$ be the basis of $B$ corresponding to that in $(2 \mathrm{~A})$ by the mirror symmetry 0.2 .2 and 0.2 .3 in Introduction.

The endomorphism of $B_{Q}$ coming from the monodromy logarithm is denoted by $N$, and the above basis is compatible with the monodromy weight filtration $M$ [ibid].

For both cases (2A) and (2B), we regard $B$ as a constant sheaf on $S^{\log }$ and also on $S$, endowed with the associated filtrations $M$.

From the asymptotics of the basis $s^{p}(0 \leq p \leq 3)$ in 2.4 , the matrix of the polarization pairings $S$ in (1A) and $Q$ in (1B) for the basis $b^{p}=s^{p}\left(u_{0}\right)$ is computed as

$$
\left(S\left(b^{p}, b^{q}\right)\right)_{p, q}=\left(Q\left(b^{p}, b^{q}\right)\right)_{p, q}=\left(\begin{array}{rrrr}
0 & 0 & 0 & -1 \\
0 & 0 & 1 & -1 \\
0 & -1 & 0 & -5 \\
1 & 1 & 5 & 0
\end{array}\right) .
$$

(3A) Sections compatible with Deligne decomposition and inducing Z-basis of $\mathrm{gr}^{M}$ for $A$-model of $V$.

Let $T^{3}, T^{2}, T^{1}$, and $T^{0}$ be the basis of $\mathcal{H}_{\mathcal{O}}^{V}$ corresponding to the $e^{3}, e^{2}, e^{1}$, and $e^{0}$ in (3B) below by the mirror symmetry 0.2 .2 and 0.2 .3 in Introduction. Then $S\left(T^{3}, T^{0}\right)=1$ and $S\left(T^{2}, T^{1}\right)=-1$. Hence $T^{3}, T^{2},-T^{0}, T^{1}$ form a symplectic base for $S$ in (1A).

Note that on $\mathrm{gr}^{M}$ they are

$$
\begin{gathered}
\operatorname{gr}_{3}^{M}\left(T^{3}\right)=1 \in H^{0}(V, \mathbf{Z}), \quad \operatorname{gr}_{2}^{M}\left(T^{2}\right)=H \in H^{2}(V, \mathbf{Z}), \\
\operatorname{gr}_{1}^{M}\left(T^{1}\right)=C \in H^{4}(V, \mathbf{Z}), \quad \operatorname{gr}_{0}^{M}\left(T^{0}\right)=[\mathrm{pt}] \in H^{6}(V, \mathbf{Z}),
\end{gathered}
$$

where $H$ and $C$ are the cohomology classes of a hyperplane section and a line on $V$, respectively. Abusing notation, we mean by $C$ the Poincaré dual class of the homology class in 2.3 .

(3B) Sections compatible with Deligne decomposition and inducing $\mathbf{Z}$-basis of $\mathrm{gr}^{\mathrm{M}}$ for $B$-model of $V^{\circ}$.

We use Deligne decomposition [4]. We consider $B$ in (2B) as a constant sheaf on $S^{\text {log }}$. We have locally free $\mathcal{O}_{S}$-submodules $\mathcal{M}_{2 p}:=\tau_{*}\left(\mathcal{O}_{S}^{\log } \otimes_{\mathrm{Z}} M_{2 p} B\right)$ and $\mathcal{F}^{p}$ in $\tau_{*}\left(\mathcal{O}_{S}^{\text {log }} \otimes_{\mathrm{Z}} B\right)=\mathcal{O}_{S} \otimes_{\mathrm{Z}} B$ (canonical extension of Deligne in Proposition 1.4.2). The mixed Hodge structure of Hodge-Tate type $(\mathcal{M}, \mathcal{F})$ 
has decomposition:

$$
\mathcal{O}_{S} \otimes_{\mathrm{Z}} B=\bigoplus_{p} I^{p, p}, \quad I^{p, p}:=\mathcal{M}_{2 p} \cap \mathcal{F}^{p} \stackrel{\sim}{\rightarrow} \mathrm{gr}_{2 p}^{\mathcal{M}} .
$$

Transporting the basis $b^{p}(0 \leq p \leq 3)$ of $B$ in (2B), regarded as sections of the constant sheaf $B$ on $S^{\text {log }}$, via isomorphism

$$
I^{p, p} \stackrel{\sim}{\rightarrow} \mathcal{O}_{S} \otimes_{\mathrm{Z}} \mathrm{gr}_{2 p}^{M} B
$$

we define sections $e^{p} \in I^{p, p}(0 \leq p \leq 3)$ over $S$. Then, $e^{3}, e^{2},-e^{0}, e^{1}$ form a symplectic basis for $Q$ in (1B), and $e^{3}=\tilde{\Omega}=\Omega / y_{0}$ over $S$.

The asymptotic relation of the $T^{p}$ in (3A) (resp. the $e^{p}$ in (3B)) can be computed, via the $s^{p}$, from (7A) (resp. (7B)) below.

(4A) A-model connection $\nabla=\nabla^{\text {even }}$ of $V$.

Let $\delta=d / d u=2 \pi i q d / d q$. The Dubrovin connection $\nabla$ (cf. [1], Sect. 8.4) is characterized by

$\nabla_{\delta} T^{0}=0, \quad \nabla_{\delta} T^{1}=T^{0}, \quad \nabla_{\delta} T^{2}=\frac{1}{(2 \pi i)^{3}} \frac{d^{3} \Phi}{d l^{3}} T^{1}=\left(5+\frac{1}{(2 \pi i)^{3}} \frac{d^{3} \Phi_{\text {hol }}}{d u^{3}}\right) T^{1}$,

$\nabla_{\delta} T^{3}=T^{2}$

$\nabla$ is flat, i.e., $\nabla^{2}=0$, and extends to a $\log$ connection over $S^{\text {log }}$.

(4B) B-model connection $\nabla=\nabla^{\mathrm{GM}}$ of $V^{\circ}$.

Let $\delta=d / d u=2 \pi i q d / d q$. The Gauss-Manin connection $\nabla$ is computed as

$\nabla_{\delta} e^{0}=0, \quad \nabla_{\delta} e^{1}=e^{0}, \quad \nabla_{\delta} e^{2}=\frac{1}{(2 \pi i)^{3}} \frac{d^{3} \Phi}{d u^{3}} e^{1}=\frac{5}{\left(1+5^{5}\right) y_{0}(z)^{2}}\left(\frac{q}{z} \frac{d z}{d q}\right)^{3} e^{1}$, $\nabla \delta e^{3} l=e^{2}$.

$\nabla$ is flat, i.e., $\nabla^{2}=0$, and extends to a $\log$ connection over $S^{\log }$.

(5A) $\nabla$-flat basis of $\mathcal{H}_{\mathrm{C}}^{V}$ inducing $\mathbf{Z}$-basis of $\mathrm{gr}^{M}$.

$$
\begin{gathered}
\tilde{s}^{0}:=T^{0}, \quad \tilde{s}^{1}:=T^{1}-u T^{0}, \quad \tilde{s}^{2}:=T^{2}-\frac{1}{(2 \pi i)^{3}} \frac{d^{2} \Phi}{d u^{2}} T^{1}+\frac{1}{(2 \pi i)^{3}} \frac{d \Phi}{d u} T^{0}, \\
\tilde{s}^{3}:=T^{3}-u T^{2}+\frac{1}{(2 \pi i)^{3}}\left(u \frac{d^{2} \Phi}{d u^{2}}-\frac{d \Phi}{d l u}\right) T^{1}-\frac{1}{(2 \pi i)^{3}}\left(u \frac{d \Phi}{d u}-2 \Phi\right) T^{0} .
\end{gathered}
$$

Then $\tilde{s}^{3}, \tilde{s}^{2},-\tilde{s}^{0}, \tilde{s}^{1}$ form a symplectic basis for $S$ in $(1 \mathrm{~A})$.

(5B) $\nabla$-flat basis for $\mathcal{H}_{\mathrm{C}}^{V^{\circ}}$ inducing $\mathrm{Z}$-basis of $\mathrm{gr}^{M}$.

$$
\begin{aligned}
& \tilde{s}^{0}:=e^{0}, \quad \tilde{s}^{1}:=e^{1}-u e^{0}, \quad \tilde{s}^{2}:=e^{2}-\frac{1}{(2 \pi i)^{3}} \frac{d^{2} \Phi}{d u^{2}} e^{1}+\frac{1}{(2 \pi i)^{3}} \frac{d \Phi}{d u} e^{0}, \\
& \tilde{s}^{3}:=e^{3}-u e^{2}+\frac{1}{(2 \pi i)^{3}}\left(u \frac{d^{2} \Phi}{d u^{2}}-\frac{d \Phi}{d u}\right) e^{1}-\frac{1}{(2 \pi i)^{3}}\left(u \frac{d \Phi}{d u}-2 \Phi\right) e^{0} .
\end{aligned}
$$

Then $\tilde{s}^{3}, \tilde{s}^{2},-\tilde{s}^{0}, \tilde{s}^{1}$ form a symplectic basis for $Q$ in (1B). 
For both cases $(5 \mathrm{~A})$ and $(5 \mathrm{~B})$, by using $(4 \mathrm{~A})$ and $(4 \mathrm{~B})$, the $\nabla$-flat bases $\tilde{s}^{p}$ are determined inductively on $0 \leq p \leq 3$ from the $T^{p}$ in (3A) and the $e^{p}$ in (3B). These $\nabla$-flat bases $\tilde{s}^{p}$ are characterized by the Frobenius solutions $y_{j}(0 \leq j \leq 3)$ in 2.2 such that $y_{0} T^{3}$ in A-model and $y_{0} e^{3}=\Omega$ in B-model coincide with

$$
y_{0} \tilde{s}^{3}+(2 \pi i)^{-1} y_{1} \tilde{s}^{2}+5(2 \pi i)^{-2} y_{2} \tilde{s}^{1}+5(2 \pi i)^{-3} y_{3} \tilde{s}^{0} .
$$

(6A), (6B) Relations of $\nabla$-flat Z-basis $s^{p}$ and the $\nabla$-flat basis $\tilde{s}^{p}$.

$s^{0}=\tilde{s}^{0}, \quad s^{1}=\tilde{s}^{1}+\tilde{s}^{0}, \quad s^{2}=\tilde{s}^{2}-\frac{5}{2} \tilde{s}^{1}+\frac{35}{12} \tilde{s}^{0}, \quad s^{3}=\tilde{s}^{3}+\frac{25}{12} \tilde{s}^{1}+\frac{25 i \zeta(3)}{\pi^{3}} \tilde{s}^{0}$.

(7A) Expression of the $T^{p}$ by the $s^{p}$ over $S^{\mathrm{log}}$.

It is computed that $T^{p}$ are written by the $\nabla$-flat $\mathbf{Z}$-basis $s^{p}$ of $\mathcal{H}_{\mathrm{Z}}^{V}$ as follows.

$$
\begin{gathered}
T^{0}=s^{0}, \quad T^{1}=s^{1}+(u-1) s^{0} \\
T^{2}=s^{2}+\left(\frac{1}{(2 \pi i)^{3}} \frac{d^{2} \Phi}{d u^{2}}+\frac{5}{2}\right) s^{1}+\left(\frac{1}{(2 \pi i)^{3}}\left(u \frac{d^{2} \Phi}{d u^{2}}-\frac{d \Phi}{d u}\right)-5 u-\frac{65}{12}\right) s^{0} \\
T^{3}=s^{3}+u s^{2}+\left(\frac{1}{(2 \pi i)^{3}} \frac{d \Phi}{d u}+\frac{5}{2} u-\frac{25}{12}\right) s^{1}+\left(\frac{1}{(2 \pi i)^{3}}\left(u \frac{d \Phi}{d u}-2 \Phi\right)\right. \\
\left.-\frac{65}{12} u+\frac{25}{12}-\frac{25 i}{\pi^{3}} \zeta(3)\right) s^{0} .
\end{gathered}
$$

(7B) Expression of the $e^{p}$ by the $s^{p}$ over $S^{\log }$.

It is computed that $e^{p}$ are written by the $\nabla$-flat $\mathbf{Z}$-basis $s^{p}$ of $\mathcal{H}_{\mathbf{Z}}^{V^{\circ}}$ as follows.

$$
\begin{gathered}
e^{0}=s^{0}, \quad e^{1}=s^{1}+(u-1) s^{0}, \\
e^{2}=s^{2}+\left(\frac{1}{(2 \pi i)^{3}} \frac{d^{2} \Phi}{d u^{2}}+\frac{5}{2}\right) s^{1}+\left(\frac{1}{(2 \pi i)^{3}}\left(u \frac{d^{2} \Phi}{d u^{2}}-\frac{d \Phi}{d u}\right)-5 u-\frac{65}{12}\right) s^{0}, \\
e^{3}=s^{3}+u s^{2}+\left(\frac{1}{(2 \pi i)^{3}} \frac{d \Phi}{d u}+\frac{5}{2} u-\frac{25}{12}\right) s^{1}+\left(\frac{1}{(2 \pi i)^{3}}\left(u \frac{d \Phi}{d u}-2 \Phi\right)\right. \\
\left.-\frac{65}{12} u+\frac{25}{12}-\frac{25 i}{\pi^{3}} \zeta(3)\right) s^{0} .
\end{gathered}
$$

(8A), (8B) Relations of integral periods and Frobenius solutions.

Let $\eta_{j}(0 \leq j \leq 3)$ be the integral periods defined by the condition that $y_{0} T^{3}$ in A-model and $y_{0} e^{3}=\Omega$ in B-model coincide with $\eta_{0} s^{3}+\eta_{1} s^{2}+\eta_{2} s^{1}+\eta_{3} s^{0}$. Then the relations in $(6 \mathrm{~A}),(6 \mathrm{~B})$ are interpreted as

$$
\begin{aligned}
& \eta_{0}=y_{0}, \quad \eta_{1}=(2 \pi i)^{-1} y_{1}, \quad \eta_{2}=5(2 \pi i)^{-2} y_{2}+\frac{5}{2}(2 \pi i)^{-1} y_{1}-\frac{25}{12} y_{0} \\
& \eta_{3}=5(2 \pi i)^{-3} y_{3}-5(2 \pi i)^{-2} y_{2}+\frac{65}{12}(2 \pi i)^{-1} y_{1}+\left(\frac{25}{12}-\frac{25 i \zeta(3)}{\pi^{3}}\right) y_{0} .
\end{aligned}
$$


Remark. The $\eta_{j}$ coincide with the corresponding coefficients of the expression of $y_{0} T^{3}$ in (7A) and of $y_{0} e^{3}=\Omega$ in (7B), and yield the same integral structure for periods given in [2] and [18]. Indeed, for the notation $w_{j}$ in [18], (8), they are related as $\varpi_{0}=\eta_{0}, \varpi_{1}=\eta_{1}, \varpi_{2}=\eta_{2}-5 \eta_{1}$, and $\varpi_{3}=-\eta_{3}-\eta_{2}-5 \eta_{1}$.

\subsection{Proofs of results in 2.5}

Proofs of $(4 A)$ and $(4 B)$ in 2.5. We prove (4B). (4A) follows by mirror symmetry theorems in Introduction 0.2 .

We improve the proof of [1], Prop. 5.6.1 carefully by a log Hodge theoretic understanding in 1.4 of the relation among a constant sheaf and the local system on $S^{\text {log }}$, the canonical extension of Deligne on $S$, and the Deligne decomposition.

We investigate the Gauss-Manin connection $\nabla$, corresponding to the local system $\mathcal{H}_{\mathrm{Z}}^{V^{\circ}}$, contracted with $\delta=2 \pi i q d / d q$. Since $e^{p}$ maps to a $\nabla\left(\mathrm{gr}_{2 p}^{\mathcal{M}}\right)$-flat element of $\operatorname{gr}_{2 p}^{\mathcal{M}}, \nabla_{\delta}\left(e^{p}\right)$ lies in $\mathcal{M}_{2 p-1}=\mathcal{M}_{2 p-2}$. But $e^{p}$ is also an element of $\mathcal{F}^{p}$, so that $\nabla_{\delta}\left(e^{p}\right)$ lies in $\mathcal{F}^{p-1}$ by Griffiths transversality. This shows that $\nabla_{\delta}\left(e^{p}\right)$ is an element of $I^{p-1, p-1}$, and it follows that

$$
\nabla_{\delta}\left(e^{3}\right)=Y_{3} e^{2}, \quad \nabla_{\delta}\left(e^{2}\right)=Y_{2} e^{1}, \quad \nabla_{\delta}\left(e^{1}\right)=Y_{1} e^{0}, \quad \nabla_{\delta}\left(e^{0}\right)=0
$$

for some $Y_{1}, Y_{2}, Y_{3} \in \mathcal{O}_{S}^{\log }$. However, since $Q\left(e^{3}, e^{1}\right)=0$ by orthogonality of Hodge filtration, we have

$$
\begin{aligned}
0 & =\delta Q\left(e^{3}, e^{1}\right)=Q\left(\nabla_{\delta}\left(e^{3}\right), e^{1}\right)+Q\left(e^{3}, \nabla_{\delta}\left(e^{1}\right)\right)=Y_{3} Q\left(e^{2}, e^{1}\right)+Y_{1} Q\left(e^{3}, e^{0}\right) \\
& =-Y_{3}+Y_{1}
\end{aligned}
$$

where the last equality follows from 2.5 (3B).

Since $\nabla$ has a regular singular point and $\delta=2 \pi i q d / d q, Y_{1}$ is holomorphic over $S$. Considering over the $\log$ point $p_{0}$, we claim $Y_{1}(0)= \pm 1$. Since $e^{1}$ is taken to be the canonical extension over $p_{0}$, we have $\nabla_{\delta}\left(e^{1}\right)=N\left(e^{1}\right)=-e^{0}$ by [17], Prop. 2.3 .4 (ii) (cf. Proposition 1.4.3). Replacing $e^{1}, e^{2}$ by $-e^{1},-e^{2}$, we have $Y_{1}(0)=1$.

Since we use the canonical coordinate $q$ in 2.2 , the arguments in [1], Sect. 5.6.4, Sect. 2.3 , yield

$$
q=\exp \left(\int Y_{1}(q) \frac{d q}{q}\right)
$$

Taking logarithm of both sides and differentiating them by $d / d \log q$, we have $Y_{1}(q)=1$, hence $\nabla_{\delta} e^{1}=e^{0}$ and $\nabla_{\delta} e^{3}=e^{2}$. Thus, relative to the basis 
$e^{0}, e^{1}, e^{2}, e^{3}$ and using the canonical coordinate $q, \nabla_{\delta}$ has the connection matrix

$$
\left(\begin{array}{cccc}
0 & 1 & & \\
& 0 & Y & \\
& & 0 & 1 \\
& & & 0
\end{array}\right)
$$

where $Y=Y_{2}$.

The proofs in [ibid, Proof of Prop. 5.6.1] for the following assertions work well: $e^{3}$ is the normalized 3-form $\tilde{\Omega}$; the Picard-Fuchs equation for $\tilde{\Omega}$ is $\nabla_{\delta}^{2}\left(\nabla_{\delta}^{2} \tilde{\Omega} / Y\right)=0 ; Y$ is the Yukawa coupling. The notation $e^{0}, e^{1}, e^{2}, e^{3}$ (resp. $\left.T^{0}, T^{1}, T^{2}, T^{3}\right)$ in the present paper corresponds to $e_{3}, e_{2}, e_{1}, e_{0}$ in [ibid, p.105] (resp. $T^{0}, T^{1}, T_{1}, T_{0}$ in [ibid, Sect. 8.5.3]).

Proofs of (5A), (5B), (6A), (6B), (7A), and (7B) in 2.5.

We use mirror symmetry theorems in Introduction 0.2 .

From $e^{p}$ in (3B) and $\nabla_{\delta}$ in (4B), we produce $\tilde{s}^{p}$ inductively on $0 \leq p \leq 3$ as in (5B). These are transported as (5A) in A-model. For the last assertion in (5B) on the relation of $\nabla$-flat basis $\tilde{s}^{p}$ and the basis of the Frobenius solutions $y_{j}$, since $y_{0} T^{3}, y_{0} e^{3}=\Omega$, and the last expression in (5B) are killed by the operator $\mathcal{L}$, it is enough to show the equality on the fiber $B_{\mathbf{C}}:=\mathbf{C} \otimes B$, i.e., the coincidence of the initial conditions. We work in A-model. By the asymptotics of the reverse relation of $(5 \mathrm{~A})$ and of the expressions of $y_{j}$ in 2.2 , we have

$$
\begin{aligned}
y_{0} T^{3}= & y_{0} \tilde{s}^{3}+y_{0} u \tilde{s}^{2}+y_{0}(2 \pi i)^{-3} \frac{d \Phi}{d u} \tilde{s}^{1}+y_{0}(2 \pi i)^{-3}\left(u \frac{d \Phi}{d u}-2 \Phi\right) \tilde{s}^{0} \\
& \sim y_{0} \tilde{s}^{3}+y_{0} u \tilde{s}^{2}+\frac{5}{2} y_{0} u^{2} \tilde{s}^{1}+\frac{5}{6} y_{0} u^{3} \tilde{s}^{0} \\
& \sim \tilde{s}^{3}+\left((2 \pi i)^{-1} \log z\right)^{2}+\frac{5}{2}\left((2 \pi i)^{-1} \log z\right)^{2} \tilde{s}^{1}+\frac{5}{6}\left((2 \pi i)^{-1} \log z\right)^{3} \tilde{s}^{0} \\
& \sim y_{0} \tilde{s}^{3}+(2 \pi i)^{-1} y_{1} \tilde{s}^{2}+5(2 \pi i)^{-2} y_{2} \tilde{s}^{1}+5(2 \pi i)^{-3} y_{3} \tilde{s}^{0} .
\end{aligned}
$$

To prove (6A), (6B), (7A), and (7B), we want to find $c^{10}, c^{21}, c^{20}, c^{32}, c^{31}, c^{30} \in$ $C$ such that, on the fiber $B$,

$$
\begin{aligned}
& \tilde{s}^{0}\left(u_{0}\right)=b^{0}, \quad \tilde{s}^{1}\left(u_{0}\right)=b^{1}+c^{10} \tilde{s}^{0}\left(u_{0}\right), \quad \tilde{s}^{2}\left(u_{0}\right)=b^{2}+c^{21} \tilde{s}^{1}\left(u_{0}\right)+c^{20} \tilde{s}^{0}\left(u_{0}\right), \\
& \tilde{s}^{3}\left(u_{0}\right)=b^{3}+c^{32} \tilde{s}^{2}\left(u_{0}\right)+c^{31} \tilde{s}^{1}\left(u_{0}\right)+c^{30} \tilde{s}^{0}\left(u_{0}\right) .
\end{aligned}
$$

Then, since $\tilde{s}^{p}$ and $s^{p}$ are $\nabla$-flat, we have

$$
s^{0}=\tilde{s}^{0}, s^{1}=\tilde{s}^{1}-c^{10} \tilde{s}^{0}, s^{2}=\tilde{s}^{2}-c^{21} \tilde{s}^{1}-c^{20} \tilde{s}^{0}, s^{3}=\tilde{s}^{3}-c^{32} \tilde{s}^{2}-c^{31} \tilde{s}^{1}-c^{30} \tilde{s}^{0} .
$$

Express the $e^{p}$ by the $s^{p}$ by using the inverse expressions of the above and of (5B). Transporting these into A-model, we get expression of the $T^{p}$ by the $s^{p}$ 
and the $c^{j k}$. Using Iritani asymptotics for the $s^{p}$ in A-model in 2.4, we get

$$
\begin{aligned}
& T^{0}= \frac{1}{5} H^{3}, \quad T^{1}=(2 \pi i)^{-1} \frac{1}{5} H^{2}+\frac{c^{10}}{5} H^{3} \\
& T^{2} \sim(2 \pi i)^{-2} H+(2 \pi i)^{-1}\left(-\frac{1}{2}+\frac{c^{21}}{5}\right) H^{2}+\left(\left(\frac{3}{2}-\frac{c^{21}}{5}+c^{10}\right) u\right. \\
&\left.+\left(\frac{7}{12}+\frac{c^{21}}{5}+\frac{c^{21} c^{10}}{5}+\frac{c^{20}}{5}\right)\right) H^{3} \\
& T^{3} \sim(2 \pi i)^{-3}+(2 \pi i)^{-2} c^{32} H+(2 \pi i)^{-1}\left(\left(-\frac{1}{2}+\frac{c^{21}}{5}-c^{32}\right) u\right. \\
&\left.+\left(\frac{5}{12}-\frac{c^{32}}{2}+\frac{c^{32} c^{21}}{5}+\frac{c^{31}}{5}\right)\right) H^{2} \\
&+\left(\left(\frac{1}{6}-\frac{c^{31}}{5}+\frac{c^{20}}{5}\right) u+\left(\frac{5 i \zeta(3)}{\pi^{3}}+\frac{c^{30}}{5}\right)\right) H^{3} .
\end{aligned}
$$

Since $T^{p} \in \mathcal{F}^{p}$ by construction ((3A), (3B) in 2.5), we see that all coefficients of $H^{j}$ in the above expressions of $T^{p}$ are zero for $j+p>3$. Thus we get $c^{10}=-1, c^{21}=5 / 2, c^{20}=-35 / 12, c^{32}=0, c^{31}=-25 / 12, c^{30}=-25 i \zeta(3) / \pi^{3}$. (6A), (6B), (7A), and (7B) follow from this and (5A), (5B).

We prove $(8 \mathrm{~A}),(8 \mathrm{~B})$. The same argument goes for both cases. We use the notation in B-model. By the definition of the $\eta_{j}$ and (5B), $\Omega=\eta_{0} s^{3}+\eta_{1} s^{2}+$ $\eta_{2} s^{1}+\eta_{3} s^{0}=y_{0} \tilde{s}^{3}+(2 \pi i)^{-1} y_{1} \tilde{s}^{2}+5(2 \pi i)^{-2} y_{2} \tilde{s}^{1}+5(2 \pi i)^{-3} y_{3} \tilde{s}^{0}$. Substituting (6B) and comparing the coefficients of $\tilde{s}^{p}$, we have expressions of the $y_{j}$ by the $\eta_{j}$. Solving these for the $\eta_{j}$, we get (8B).

Remark. It was pointed out by Hiroshi Iritani that the definitions and the descriptions of integral structures 3.5, 3.6 in [30] are insufficient. Actually, they were the first approximations of integral structures by means of $\mathrm{gr}^{M}$, which are characterized by the Frobenius solutions as in the last statement of (5A) and (5B) in 2.5. The second proof in [ibid, 3.9] works well even in this approximation.

\section{Proof of Theorem 0.4.1}

In this section, we prove Theorem 0.4.1 in Introduction for open mirror symmetry of quintic threefolds. We prove it by constructing a normal function in log mixed Hodge theory for B-model in 3.1-3.2 below. This argument is applicable to the case of A-model by the theorems in Introduction 0.2 and 0.3 and the correspondence table in 2.5. We give some discussions on geometries and local systems in 3.3. 


\subsection{Proof of Theorem 0.4.1 over log disc $S$}

We consider B-model. To make the monodromy of $\mathcal{T}_{B}$ unipotent, we take a double cover $z^{1 / 2} \mapsto z$. Let $S$ be a neighborhood disc of $p_{0}$ in the $z^{1 / 2}$-plane endowed with $\log$ structure associated to the divisor $p_{0}$ in $S$. Denote by $\mathcal{H}$ and $\mathcal{T}$ the pullbacks of the log Hodge structure $\mathcal{H}^{V^{\circ}}$ and the tension $\mathcal{T}_{B}$ by the double covering, respectively.

We are looking for an extension $\tilde{\mathcal{H}}$ :

$$
0 \rightarrow \mathcal{H} \rightarrow \tilde{\mathcal{H}} \rightarrow \mathbf{Z}(-2) \rightarrow 0
$$

of log mixed Hodge structures with $\operatorname{gr}_{4}^{W} \tilde{\mathcal{H}}=\mathbf{Z}(-2)$ and $\operatorname{gr}_{3}^{W} \tilde{\mathcal{H}}=\mathcal{H}$, which has liftings $1_{\mathbf{Z}}$ and $1_{F}$ of $(2 \pi i)^{-2} \cdot 1 \in \mathbf{Z}(-2)$ in $\tilde{\mathcal{H}}$ respecting the lattice and the Hodge filtration, respectively, such that the tension $\mathcal{T}$ is described as

$$
Q\left(1_{F}-1_{\mathbf{z}}, \Omega\right)=\int_{C_{-}}^{C_{+}} \Omega=\mathcal{T},
$$

where $Q$ is the polarization of $\mathcal{H}$ coming from 2.5 (1B) and $\Omega$ is the 3 -form from 2.2.

To find such a log mixed Hodge structure, we use the basis $e^{p}(0 \leq p \leq 3)$ respecting the Deligne decomposition of $(\mathcal{H}, \mathcal{M}, \mathcal{F})$ from $2.5(3 \mathrm{~B})$, and the $\nabla$-flat integral basis $s^{p}(0 \leq p \leq 3)$ from $2.5(5 \mathrm{~B})$. We also use the integral periods $\eta_{j}(0 \leq j \leq 3)$ in $2.5(8 \mathrm{~B})$. Note that these players are already extended and live together over $S^{\log }$.

Let the local system $L_{\mathbf{Q}}$ and the Néron model $J_{L_{\mathbf{Q}}}$ be as in 0.4 in Introduction (see also 1.8). Then $J_{L_{\mathbf{Q}}}=\mathcal{E} x t_{\mathrm{LMH} / S}^{1}(\mathbf{Z}(-2), \mathcal{H})$, and let $1_{\mathbf{Z}}:=\left((2 \pi i)^{-2} \cdot 1\right.$, $\left.-\left(\mathcal{T} / \eta_{0}\right) s^{0}\right) \in \tilde{\mathcal{H}}_{\mathbf{Z}}$ be a lifting of $(2 \pi i)^{-2} \cdot 1 \in \mathbf{Z}(-2)=\left(\mathrm{gr}_{4}^{W}\right)_{\mathbf{Z}}$, where $\left(\mathcal{T} / \eta_{0}\right) s^{0} \in \mathcal{H}_{\mathcal{O}^{\text {log }}}=\left(\mathrm{gr}_{3}^{W}\right)_{\mathcal{O}^{\text {log }}}$. In particular, the connection $\nabla=\nabla^{\mathrm{GM}}$ on $\mathcal{H}$ is extended over $\tilde{\mathcal{H}}$ by $\nabla\left(1_{\mathrm{Z}}\right)=0$.

To find $1_{F}$, we write $1_{F}-1_{\mathbf{z}}=a e^{3}+b e^{2}+c e^{1}-\left(\mathcal{T} / \eta_{0}\right) e^{0}$ with $a, b, c \in \mathcal{O}_{S}^{\log }$ by using (1). The Griffiths transversality condition on $1_{F}-1_{Z}$ is understood as vanishing of the coefficient of $e^{0}$ in $\nabla_{\delta}\left(1_{F}-1 \mathrm{z}\right)$. Using $2.5(4 \mathrm{~B})$, we have

$$
\nabla_{\delta}\left(1_{F}-1_{\mathbf{Z}}\right)=(\delta a) e^{3}+(a+\delta b) e^{2}+\left(b \frac{1}{(2 \pi i)^{3}} \frac{d^{3} \Phi}{d u^{3}}+\delta c\right) e^{1}+\left(c-\delta\left(\mathcal{T} / \eta_{0}\right)\right) e^{0}
$$

Hence, the above condition is equivalent to $c=\delta\left(\mathcal{T} / \eta_{0}\right)$ and $a, b$ arbitrary. Using the relation "modulo $F^{2}$," we can take $a=b=0$. Thus

$$
1_{F}=1_{\mathbf{z}}+\left(\delta\left(\mathcal{T} / \eta_{0}\right)\right) e^{1}-\left(\mathcal{T} / \eta_{0}\right) e^{0} .
$$

The pair $1_{\mathbf{Z}}$ and $1_{F}$ yields the desired element of $\mathcal{E}_{x t_{\mathrm{LMH} / S}^{1}}(\mathbf{Z}(-2), \mathcal{H})$, hence $1_{F}-1_{\mathrm{Z}}$ yields the desired log normal function. Theorem 0.4.1 (1) is proved.

Next, we will find a splitting of the weight filtration $W$ of the local system $L_{\mathbf{Q}}$, i.e., a splitting of $W$ which is compatible with the local monodromy of 
the local system $L_{Q}$. We use the monodromy table (3.14) in [31]. This is computed for A-model but applicable also for B-model by the theorems in Introduction $0.2-0.3$ and in Section 2.5. Let $T_{\infty}^{2}$ be the monodromy around $p_{0}: z^{1 / 2}=0$ and put $N:=\log \left(T_{\infty}^{2}\right)$. By [ibid], $N\left(\mathcal{T} / \eta_{0}\right)=-1$ hence $N\left(1_{\mathbf{z}}\right)=s^{0}$. On the other hand, we have $N\left(s^{1}\right)=-2 s^{0}$. (Here we use the rotation of the monodromy as $\log z \mapsto \log z+2 \pi i$.) Define

$$
1_{\mathbf{Z}}^{\mathrm{spl}}:=1_{\mathbf{Z}}+\frac{1}{2} s^{1}=\left((2 \pi i)^{-2} \cdot 1, \frac{1}{2} s^{1}-\frac{\mathcal{T}}{\eta_{0}} s^{0}\right) \in \tilde{\mathcal{H}}_{\mathbf{Q}} .
$$

Then $N\left(1_{\mathbf{Z}}^{\mathrm{spl}}\right)=0$, and this gives the desired splitting of $W$ of the local system $L_{Q}$.

A lifting $1_{F}^{\mathrm{spl}}$ for $1_{\mathbf{Z}}^{\mathrm{spl}}$, respecting the Hodge filtration, is computed as before and we get

$$
1_{F}^{\mathrm{spl}}=1_{\mathbf{Z}}^{\mathrm{spl}}+\left(\delta\left(\frac{\mathcal{T}}{\eta_{0}}\right)\right) e^{1}-\frac{\mathcal{T}}{\eta_{0}} e^{0} .
$$

The pair $1_{\mathbf{Z}}^{\text {spl }}$ and $1_{F}^{\text {spl }}$ yields the desired element of $\mathcal{E} x t_{\mathrm{LMH} / S}^{1}(\mathbf{Z}(-2), \mathcal{H})$ which splits the weight filtration $W$ of the local system $L_{\mathbf{Q}}$. Note that $1_{F}^{\mathrm{spl}}-1_{\mathrm{Z}}^{\mathrm{spl}}=$ $1_{F}-1_{\mathbf{Z}}=\left(\delta\left(\mathcal{T} / \eta_{0}\right)\right) e^{1}-\left(\mathcal{T} / \eta_{0}\right) e^{0}$. Theorem 0.4.1 (2) is proved.

Theorem 0.4.1 (3) follows immediately from the above results.

We add a remark that the $W$-relative $N$-filtration $M=M(N, W)$ on $H_{\mathbf{R}}$ in the admissibility condition 1.5 (1) is given by

$$
\begin{aligned}
& M_{-1}=0 \subset M_{0}=M_{1}=\mathbf{R} s^{0} \subset M_{2}=M_{3}=M_{1}+\mathbf{R} s^{1} \\
& \subset M_{4}=M_{5}=M_{3}+\mathbf{R} s^{2}+\mathbf{R} 1_{\mathbf{Z}} \subset M_{6}=\tilde{\mathcal{H}}_{\mathbf{R}}=M_{5}+\mathbf{R} s^{3} .
\end{aligned}
$$

\subsection{Proofs of (1) and (2) in Theorem 0.4.1 over log point $p_{0}$}

We still consider B-model. We show here that (1) and (2) in Theorem 0.4.1 have meanings just over the $\log$ point $p_{0}$ and that the computations in their proofs become simpler.

Recall that

$$
\mathcal{T}=-\frac{\eta_{1}}{2}-\frac{\eta_{0}}{4}+a_{0} \tau \quad\left(a_{0}:=\frac{15}{\pi^{2}}, \tau: \text { tau function }\right)
$$

from [31]. We substitute $z^{1 / 2}=0$ to $\mathcal{T}$ carefully as follows. Recall $\eta_{1}=\eta_{0} u$ from 2.2 and $u=x+i y$ from 2.4. Write $v:=x+i \infty$ and define

$$
\mathcal{T}(0):=-\frac{v}{2}-\frac{1}{4}+a_{0} \quad \text { in } \mathcal{O}_{p_{0}}^{\log }=\mathbf{C}[v]
$$

We abuse the notation $e^{p}$ and $s^{p}$ also for their restrictions over the log point $p_{0}$, and so they live together over $p_{0}^{\log }=\left(\mathbf{S}^{1}, \mathbf{C}[v]\right)$. 
Similarly as in 0.4 in Introduction, but using now $\mathcal{T}(0) s^{0}$ instead of $\left(\mathcal{T} / \eta^{0}\right) s^{0}$ because $\eta^{0}(0)=1$, we define a local system $L_{\mathbf{Q}}$ and a Néron model $J_{L_{\mathbf{Q}}}$ lying over $L_{\mathbf{Q}}$. Let $\tilde{\mathcal{H}}$ be an extension of log mixed Hodge structures over the $\log$ point $p_{0}$, we are looking for, like in 3.1 , and let $1 \mathrm{z}:=\left((2 \pi i)^{-2}\right.$. $\left.1,-\mathcal{T}(0) s^{0}\right)$ be a lifting of $(2 \pi i)^{-2} \cdot 1 \in \mathbf{Z}(-2)=\left(\operatorname{gr}_{4}^{W}\right) \mathbf{Z}$ in $\tilde{\mathcal{H}}_{\mathbf{Z}}$. Hence the connection $\nabla$ on $\mathcal{H}$ is extended over $\tilde{\mathcal{H}}$ by $\nabla\left(1_{\mathbf{z}}\right)=0$. Note that both $2 \pi i q \frac{d}{d q}$ and $2 \pi i z \frac{d}{d z}$ coincide with $\frac{d}{d v}$ now, which is denoted by $\delta$. To find $1_{F}$, write $1_{F}-1_{\mathbf{Z}}=a e^{3}+b e^{2}+c e^{1}-\mathcal{T}(0) e^{0}\left(a, b, c \in \mathbf{C}[v], \eta_{0}(0)=1\right)$ and compute $\nabla_{\delta}\left(1_{F}-1_{\mathbf{Z}}\right)$ as in 3.1. Then, by the Griffiths transversality, we have $c=-1 / 2$, $a$ and $b$ arbitrary. By the relation "modulo $F^{2}$," $a$ and $b$ can be reduced to 0 . Thus, we have

$$
1_{F}=1_{\mathbf{Z}}+(\delta \mathcal{T}(0)) e^{1}-\mathcal{T}(0) e^{0}=1_{\mathbf{z}}-\frac{1}{2} e^{1}+\left(\frac{v}{2}+\frac{1}{4}-a_{0}\right) e^{0} .
$$

The pair $1_{\mathbf{Z}}$ and $1_{F}$ yields the desired element of $\mathcal{E} x t_{\mathrm{LMH} / \mathcal{S}}^{1}(\mathbf{Z}(-2), \mathcal{H})$. Theorem 0.4.1 (1) is proved.

The splitting of the weight filtration $W$ of the local system $L_{\mathbf{Q}}$ is computed as in 3.1 but more simply, and we define

$$
1_{\mathbf{Z}}^{\text {spl }}:=1_{\mathbf{Z}}+\frac{1}{2} s^{1}=\left((2 \pi i)^{-2} \cdot 1, \frac{1}{2} s^{1}-\mathcal{T}(0) s^{0}\right) \in \tilde{\mathcal{H}}_{\mathbf{Q}} .
$$

Similarly, a lifting $1_{F}^{\text {spl }}$ for $1_{Z}^{\text {spl }}$ is computed simply, and we get

$$
1_{F}^{\mathrm{spl}}=1_{\mathrm{Z}}^{\log }+(\delta \mathcal{T}(0)) e^{1}-\mathcal{T}(0) e^{0} .
$$

Theorem 0.4.1 (2) is proved.

Remark. Note that 0.4.1 (3) does not have meaning in the present context. This is because tau function disappears except its constant term when $z^{1 / 2}=0$ is substituted. That is, in this step, we lose the transcendental data of the tension $\mathcal{T}$, contained as the extension of its underlying local system, from which we can recover the position of the quintic mirror in its complex moduli space.

\subsection{Discussions on geometries and local systems}

We discuss here the relation with geometries and local systems considered in [31] and [23]. Forgetting Hodge structures, we consider only local systems corresponding to the monodromy of integral periods and tensions.

Let $V_{\psi}$ and $V_{\psi}^{\circ}$ be a quintic threefold and its mirror from 2.1. Let $S$ be a small neighborhood in the $z$-plane ( $z$ in 2.2) of the maximal unipotent monodromy point $p_{0}$ endowed with the log structure associated to the divisor $p_{0}$.

We first consider B-model. Let the setting be as in [23], Sect. 4. For $z \neq 0$ near 0 , i.e., near $p_{0}$, let $V_{z}^{\circ}$ be the mirror quintic and $C_{+, z} \cup C_{-, z}$ be the disjoint union of smooth rational curves on $V_{z}^{\circ}$ coming from the two conics contained 
in $V_{\psi} \cap\left\{x_{1}+x_{2}=x_{3}+x_{4}=0\right\} \subset \mathbf{P}^{4}(\mathbf{C})$. From the relative homology sequence for $\left(V_{z}^{\circ},\left(C_{+, z} \cup C_{-, z}\right)\right)$, we have

$$
\text { (1) } 0 \rightarrow H_{3}\left(V_{z}^{\circ} ; \mathbf{Z}\right) \rightarrow H_{3}\left(V_{z}^{\circ},\left(C_{+, z} \cup C_{-, z}\right) ; \mathbf{Z}\right) \stackrel{\circ}{\rightarrow} \mathbf{Z}\left(\left[C_{+, z}\right]-\left[C_{-, z}\right]\right) \rightarrow 0 \text {, }
$$

where $\mathbf{Z}\left(\left[C_{+, z}\right]-\left[C_{-, z}\right]\right)$ is $\operatorname{Ker}\left(H_{2}\left(C_{+, z} \cup C_{-, z} ; \mathbf{Z}\right) \rightarrow H_{2}\left(V_{z}^{\circ} ; \mathbf{Z}\right)\right)$. The monodromy $T_{\infty}$ around $p_{0}$ interchanges $C_{+, z}$ and $C_{-, z}$.

Respecting the sequence (1), we take a family of cycles Poincaré duality isomorphic to the flat integral basis $s^{p}(0 \leq p \leq 3)$ in 2.4 and a family of chains joining from $C_{-, z}$ to $C_{+, z}$ (a choice up to integral cycles and up to half twists), and over them integrate the family of 3-forms $\Omega(z)$ with $\log$ pole over $z=0$ ( $z$ in the punctured disc in the $z$-plane) in 2.2 , then we have a family of vectors $\left(\eta_{0}, \eta_{1}, \eta_{2}, \eta_{3}, \mathcal{T}\right)$ consisting of periods $(2.5(8 \mathrm{~B}))$ and a tension. This corresponds to the data in [31], [23] (cf. Remark in 2.5). Since $T_{\infty}(\mathcal{T})=-\left(\mathcal{T}+\eta_{1}+\eta_{0}\right)$ by [31], (3.14), we find $\mathcal{T}+\frac{1}{2} \eta_{1}+\frac{1}{4} \eta_{0}=\frac{15}{\pi^{2}} \tau$ (see 3.2 (1)) is an eigenvector of the monodromy $T_{\infty}$ with eigenvalue -1 .

The family of sequences $(1)(z \neq 0)$ forms an exact sequence of local systems of $\mathbf{Z}$-modules. Pulling this back to $S^{*}$ in 3.1 by the double cover $z^{1 / 2} \mapsto z$, we have a sequence with unipotent local monodromy and its extension over $S^{\log }$. Applying Tate twist $(-3)$ and Poincaré duality isomorphism to the left and the right ends of this exact sequence, we have a local system $L^{\prime}$ over $S^{\log }$ which is an extension of $\mathbf{Z}(-2)$ by $\mathcal{H}_{\mathbf{Z}}$ :

$$
0 \rightarrow \mathcal{H}_{\mathbf{Z}} \rightarrow L^{\prime} \rightarrow \mathbf{Z}(-2) \rightarrow 0 .
$$

Take a lifting $1_{\mathbf{Z}}:=\left((2 \pi i)^{-2} \cdot 1,\left(\mathcal{T} / \eta_{0}\right) s^{0}\right)$ in $L^{\prime}$ of $(2 \pi i)^{-2} \cdot 1 \in \mathbf{Z}(-2)$, and extend $\nabla$ on $\mathcal{H}_{\mathbf{Z}}$ over $L^{\prime}$ by $\nabla\left(1_{\mathbf{Z}}\right)=0$. We look for a $\nabla$-flat $T_{\infty}^{2}$-invariant element associated to $1_{\mathbf{Z}}$. This is done as in 3.1 , and we get $1_{\mathbf{Z}}^{\mathrm{spl}}:=1_{\mathbf{Z}}-\left(s^{1} / 2\right)$. Thus we know that $L^{\prime}$ coincides with $\tilde{\mathcal{H}}_{\mathrm{z}}$ in 3.1 .

For the relative monodromy weight filtration $M=M(N, W)$ of $L^{\prime}$, we see that $1_{\mathrm{Z}} \in M_{4}$ and $s^{1} \in M_{2}$ are the smallest filters containing each element in question. Taking the graded quotients by $M$ of the sequence (2), we have

$$
\begin{gathered}
\operatorname{gr}_{6}^{M} \mathcal{H}_{\mathrm{Z}} \stackrel{\sim}{\rightarrow} \mathrm{gr}_{6}^{M} L^{\prime}, \\
0 \rightarrow \mathrm{gr}_{4}^{M} \mathcal{H}_{\mathrm{Z}} \rightarrow \mathrm{gr}_{4}^{M} L^{\prime} \rightarrow \mathrm{Z}(-2) \rightarrow 0, \\
0 \rightarrow \mathrm{gr}_{2}^{M} \mathcal{H}_{\mathbf{Z}} \rightarrow \mathrm{gr}_{2}^{M} L^{\prime} \rightarrow(\text {-torsion }) \rightarrow 0, \\
\operatorname{gr}_{0}^{M} \mathcal{H}_{\mathbf{Z}} \stackrel{\sim}{\rightarrow} \mathrm{gr}_{0}^{M} L^{\prime} .
\end{gathered}
$$

Here we abuse the notation $M$ also for the monodromy filtration on $\mathcal{H}_{\mathbf{z}}$, because it coincides with the restriction of $M=M(N, W)$ to $\mathcal{H}_{\mathrm{z}}$. The 2-torsion in the third sequence of (3) corresponds to a half twist of chains from $C_{-}$to $C_{+}$. Standing on a half integral point and looking at the integral points nearby, we have two orientations. These correspond to the two orientations of a half 
twist of the chains, and also correspond to $\mathcal{T}_{ \pm}:= \pm\left(\frac{15}{\pi^{2}} \tau-\frac{\eta_{0}}{4}\right)-\frac{\eta_{1}}{2}$ in [31]. $\mathcal{T}_{-}$is different from $-\mathcal{T}_{+}$by the complementary half twist, i.e., $\mathcal{T}_{+}+\mathcal{T}_{-}=-\eta_{1}$.

By using mirror symmetries in $0.2-0.4$, or more precisely, by the results in Section 2.5 and Section $3.1, \mathcal{H}_{\mathbf{Z}}=\mathcal{H}_{\mathbf{Z}}^{V^{\circ}}, \mathcal{T}=\mathcal{T}_{B}, 1_{\mathbf{Z}}, \nabla=\nabla^{\mathrm{GM}}, 1_{\mathbf{Z}}^{\mathrm{spl}}$, and $M=M(N, W)$ of B-model are transformed to the corresponding $\mathcal{H}_{\mathbf{Z}}=\mathcal{H}_{\mathbf{Z}}^{V}$, $\mathcal{T}=y_{0} \mathcal{T}_{A}, 1_{\mathbf{Z}}, \nabla=\nabla^{\text {even }}, 1_{\mathbf{Z}}^{\text {spl }}$, and $M=M(N, W)$ of A-model, and the exact sequences (2) and (3) of B-model are transformed to the corresponding exact sequences of A-model.

It is interesting to study the relations of these exact sequences with the geometries of Fermat quintic $V=V_{\psi}$ with $\psi=0$ and its Lagrangian submanifold $L g:=V \cap \mathbf{P}^{4}(\mathbf{R})$ in [31], 2.1; [23], 3.

\section{Remark.}

(1) The argument in 3.3 can be performed even over the log point $p_{0}$.

(2) [26] and [19] are related with the topics in this subsection.

\section{References}

[1] D. A. Cox, S. KATZ, Mirror symmetry and algebraic geometry MathSirveys and Monographs 68, AMS, 1999, pp. 469. MR 2000d:14048.

[2] P. Candelas, C. De la Ossa, P. S. Green, L. Parks, A pair of Calabi-Yau manifolds as an exactly soluble superconformal theory, Nuclear Physics B 358 (1991), 21-74.

[3] P. DELIGNE, Équations differentielles à points singuliers réguliers Lect. Notes in Math. No. 163, Springer-Verlag, 1970, MR 54\#5232.

[4] L Local behavior of Hodge structures at infinity, in Mirror Symmetry II (B. Greene and S.-T. Yau, eds.), AMS/IP Stud. Adv. Math. 1, 1997, 683-699. MR 98a:14015.

[5] A. B. Givental, Equivariant Gromov-Witten invariants, Internat. Math. Res. Notes 13 (1996), 613-663.

[6] H. IRITANI, An integral structure in quantum cohomology, Adv. Math. 222 (3) (2009), 1016-1079.

[7] _ Quantum cohomology and periods, Ann. Inst. Fourier (Grenoble) 61 no.7 (2011), 2909 - 2958.

[8] K. Kato, C. NA KaYAma, Log Betti cohomology, log étale cohomology, and log de Rham cohomology of log schemes over C, Kodai Math. J. 22 (1999), 161-186. MR 2000i:14023.

[9] K. KaTO, C. NAKAYAMA, S. Usui, SL(2)-orbit theorem for degeneration of mixed Hodge structure, J. Algebraic Geometry 17 (2008), 401-479. MR 2009b:14020.

[10] _ Classifying spaces of degenerating mixed Hodge structures, I: Borel-Serre spaces, Advanced Studies in Pure Math. 54: Algebraic Analysis and Around, 2009, 187-222. MR 2010g:14010.

[11] — Log intermediate Jacobians, Proc. Japan Acad. 86, Ser. A (2010), 73-78. 
[12] , Classifying spaces of degenerating mixed Hodge structures, II: Spaces of SL(2)-orbits, Kyoto J. Math. 51-1: Nagata Memorial Issue (2011), 149-261. MR 2012f:14012.

[13] __ Classifying spaces of degenerating mixed Hodge structures, III: Spaces of nilpotent orbits, J. Algebraic Geometry, 22 (2013), 671-772.

[14] _ Néron models for admissible normal functions, Proc. Japan Acad. 90, A (2014), 6-10.

[15] K. KATO, S. UsUI, Logarithmic Hodge structures and classifying spaces (summary), in CRM Proc. and Lect. Notes: The Arithmetic and Geometry of Algebraic Cycles, (NATO Advanced Study Institute / CRM Summer School 1998: Banff, Canada), 24 (1999), 115-130. MR 2001e:14009

[16] _ Borel-Serre spaces and spaces of SL(2)-orbits, Advanced Studies in Pure Math. 36: Algebraic Geometry 2000, Azumino, 2002, 321-382. MR 2004f:14021.

[17] _ Classifying spaces of degenerating polarized Hodge structures, Ann. Math. Studies, Princeton Univ. Press 169, 2009, pp. 288. MR 2009m:14012.

[18] G. LAPORTE, J. WALCHER, Monodromy of an inhomogenoues Picard-Fuchs equation, SIGMA 8 (2012), 056, 10 pages.

[19] S. LI, B. LIAN, S.-T. YAU, Picard-Fuchs equations for relative periods and Abel-Jacobi map for Calabi-Yau hypersurfaces, Amer. J. Math. 134-5 (2012), 1345-1384. MR 2975239.

[20] B. LiAn, K. LIU, S.-T. YAU, Mirror principle I, Asian J. Math. 1 (1997), 729-763. MR 99e: 14062.

[21] D. MORRISON, Mirror symmetry and rational curves on quintic threefolds: $A$ guide for mathematicians, J. of AMS 6-1 (1993), 223-247. MR 93j: 14047.

[22] _ Mathematical aspects of mirror symmetry, in Complex algebraic geometry (Park City, UT, 1993), IAS/Park City Math. Ser. 3 (1997), 265-327. MR 98g: 14044.

[23] D. MORRISON, J. WAlChER, D-branes and normal functions, Adv. Theor. Math. Phys. 13-2 (2009), 553-598. MR 2010b: 14081.

[24] A. Ogus, On the logarithmic Riemann-Hilbert correspondences, Documenta Math. Extra volume: Kazuya Kato's Fiftieth birthday, 2003, 655-724.

[25] R. PANDHARIPANDE, Rational curves on hypersurfaces [after A. Givental], Séminaire Bourbaki 848, Astérisque 252 (1998), Exp. No. 848, 5, 307-340. MR 2000e:14094.

[26] R. Pandharipande, J. Solomon, J. Walcher, Disk enumeration on the quintic 3-fold, J. Amer. Math. Soc. 21-4 (2008), 1169-1209. MR 2009j:14075.

[27] W. SCHMID, Variation of Hodge structure: The singularities of the period mapping, Invent. Math. 22 (1973), 211-319. MR 52甘3157.

[28] S. USUI, Variation of mixed Hodge structure arising from family of logarithmic deformations II: Classifying space, Duke Math. J. 51-4 (1984), 851-875. MR 86h:14005.

[29] __ Generic Torelli theorem for quintic-mirror family, Proc. Japan Acad. 84, A (2008), 143-146. MR 2010b: 14012.

[30] __ A study of mirror symmetry through log mixed Hodge theory, Hodge Theory, Complex Geometry, and Representation Theory, Contemporary Math., AMS 608 (2014), 285-311. 
[31] J. WALCHER, Opening mirror symmetry on the quintic, Commun. Math. Phys. 276 (2007), 671-689. MR 2008m:14111.

\section{Sampei USUI}

Graduate School of Science

Osaka University

Toyonaka, Osaka, 560-0043, Japan

usui@math.sci.osaka-u.ac.jp 